\title{
Graded Limits of Minimal Affinizations in Type $D^{\star}$
}

Katsuyuki NAOI

Institute of Engineering, Tokyo University of Agriculture and Technology,

3-8-1 Harumi-cho, Fuchu-shi, Tokyo, Japan

E-mail: naoik@cc.tuat.ac.jp

Received October 30, 2013, in final form April 14, 2014; Published online April 20, 2014

http://dx.doi.org/10.3842/SIGMA.2014.047

\begin{abstract}
We study the graded limits of minimal affinizations over a quantum loop algebra of type $D$ in the regular case. We show that the graded limits are isomorphic to multiple generalizations of Demazure modules, and also give their defining relations. As a corollary we obtain a character formula for the minimal affinizations in terms of Demazure operators, and a multiplicity formula for a special class of the minimal affinizations.
\end{abstract}

Key words: minimal affinizations; quantum affine algebras; current algebras

2010 Mathematics Subject Classification: 17B37; 17B10

\section{Introduction}

Let $\mathfrak{g}$ be a complex simple Lie algebra, $\mathbf{L} \mathfrak{g}=\mathfrak{g} \otimes \mathbb{C}\left[t, t^{-1}\right]$ the associated loop algebra, and $U_{q}(\mathbf{L} \mathfrak{g})$ the quantum loop algebra. In [1], Chari introduced an important class of finite-dimensional simple $U_{q}(\mathbf{L} \mathfrak{g})$-modules called minimal affinizations. For a simple $U_{q}(\mathfrak{g})$-module $V$, we say a simple $U_{q}(\mathbf{L} \mathfrak{g})$-module $\widehat{V}$ is an affinization of $V$ if the highest weight of $\widehat{V}$ is equal to that of $V$. One can define a partial ordering on the equivalence classes (the isomorphism classes as a $U_{q}(\mathfrak{g})$-module) of affinizations of $V$, and modules belonging to minimal classes are called minimal affinizations (a precise definition is given in Section 2.6). For example, a Kirillov-Reshetikhin module is a minimal affinization whose highest weight is a multiple of a fundamental weight. Minimal affinizations have been the subjects of many articles in the recent years. See [7, 10, 12, 18, 19, 21] for instance. For the original motivations of considering minimal affinizations, see [1, Introduction]. Given a minimal affinization, one can consider its classical limit. By restricting it to the current algebra $\mathfrak{g}[t]=\mathfrak{g} \otimes \mathbb{C}[t]$ and taking a pull-back, a graded $\mathfrak{g}[t]$-module called graded limit is obtained. Graded limits are quite important for the study of minimal affinizations since the $U_{q}(\mathfrak{g})$-module structure of a minimal affinization is completely determined by the $U(\mathfrak{g})$-module structure of its graded limit.

Graded limits of minimal affinizations were first studied in $[2,5]$ in the case of KirillovReshetikhin modules, and subsequently the general ones were studied in [18]. In that paper, Moura presented several conjectures for the graded limits of minimal affinizations in general types, and partially proved them. Graded limits of minimal affinizations in type $A B C$ were further studied in [21]. In that paper the author proved that the graded limit of a minimal affinization in these types is isomorphic to a certain $\mathfrak{g}[t]$-module $D\left(w_{\circ} \xi_{1}, \ldots, w_{\circ} \xi_{n}\right)$. Here $w_{\circ}$ is the longest element of the Weyl group of $\mathfrak{g}, \xi_{j}$ are certain weights of the affine Lie algebra $\widehat{\mathfrak{g}}$ which are $\mathfrak{g}$-dominant, and $D\left(w_{\circ} \xi_{1}, \ldots, w_{\circ} \xi_{n}\right)$ is a $\mathfrak{g}[t]$-submodule of a tensor product of simple highest weight $\widehat{\mathfrak{g}}$-modules, which is generated by the tensor product $v_{w_{\circ} \xi_{1}} \otimes \cdots \otimes v_{w_{\circ} \xi_{n}}$ of the extremal weight vectors with weights $w_{\circ} \xi_{j}$. As a corollary of this, a character formula for minimal affinizations was given in terms of Demazure operators. In addition, the defining relations of graded limits conjectured in [18] were also proved.

\footnotetext{
${ }^{\star}$ This paper is a contribution to the Special Issue on New Directions in Lie Theory. The full collection is available at http://www.emis.de/journals/SIGMA/LieTheory2014.html
} 
In type $A B C$ a minimal affinization with a fixed highest weight is unique up to equivalence, and the graded limit of a minimal affinization depends only on the equivalence class. As a consequence, the module $D\left(w_{\circ} \xi_{1}, \ldots, w_{\circ} \xi_{n}\right)$ can be determined from the highest weight only. (If the highest weight is $\lambda=\sum_{1 \leq i \leq n} \lambda_{i} \varpi_{i}$ where $\varpi_{i}$ are fundamental weights, then $\xi_{j}$ are roughly equal to $\lambda_{i}\left(\varpi_{i}+a_{i} \Lambda_{0}\right)$ where $\Lambda_{0}$ is the fundamental weight of $\widehat{\mathfrak{g}}$ associated with the distinguished node 0 , and $a_{i}=1$ if the simple root $\alpha_{i}$ is long and $a_{i}=1 / 2$ otherwise. For the more precise statement, see [21].)

In contrast to this, in type $D$ there are nonequivalent minimal affinizations with the same highest weights. It was proved in [10], however, that even in type $D$ if the given highest weight satisfies some mild condition (see Section 2.6), then there are at most 3 equivalence classes of minimal affinizations with the given highest weight. We say a minimal affinization is regular if its highest weight satisfies this condition. The purpose of this paper is to study the graded limits of regular minimal affinizations of type $D$ using the methods in [21].

In the sequel we assume that $\mathfrak{g}$ is of type $D_{n}$. Let $\boldsymbol{\pi}$ be Drinfeld polynomials and assume that the simple $U_{q}(\mathbf{L} \mathfrak{g})$-module $L_{q}(\boldsymbol{\pi})$ associated with $\boldsymbol{\pi}$ is a regular minimal affinization. Then in a certain way we can associate with $L_{q}(\boldsymbol{\pi})$ a vertex $s \in\{1, n-1, n\}$ of the Dynkin diagram of $\mathfrak{g}$ (see Section 2.6). In the case where the number of equivalence classes are exactly 3 , this $s$ parameterizes the equivalence class of $L_{q}(\boldsymbol{\pi})$. In this paper we show that there exists a sequence $\xi_{1}^{(s)}, \ldots, \xi_{n}^{(s)}$ of $\mathfrak{g}$-dominant $\widehat{\mathfrak{g}}$-weights such that the graded limit $L(\boldsymbol{\pi})$ of $L_{q}(\boldsymbol{\pi})$ is isomorphic to $D\left(w_{\circ} \xi_{1}^{(s)}, \ldots, w_{\circ} \xi_{n}^{(s)}\right)$ (Theorem 3.1). Here $\xi_{j}^{(s)}$ depends not only on the highest weight of $L_{q}(\boldsymbol{\pi})$ but also $s$, and the correspondence is less straightforward compared with the case of type $A B C$ (see Section 3.1 for the precise statement). As a consequence, we give a character formula for $L_{q}(\boldsymbol{\pi})$ in terms of Demazure operators (Corollary 3.5). We also prove the defining relations of the graded limits $L(\boldsymbol{\pi})$ conjectured in [18] (Theorem 3.2), which also depends not only on the highest weight but also $s$.

Recently Sam proved in [22] some combinatorial identity in type $B C D$, and gave a multiplicity formula for minimal affinizations in type $B C$ using the identity and results in [4] and [21]. By applying the identity of type $D$ to our results, we also obtain a similar multiplicity formula for a special class of minimal affinizations in type $D$, which gives multiplicities in terms of the simple Lie algebra of type $C$ (Corollary 3.8 ).

The proofs of most results are similar to those in [21] and are in some respects even simpler since the type $D$ is simply laced. For example we do not need the theory of $q$-characters, which was essentially needed in loc. cit.

The organization of the paper is as follows. In Section 2, we give preliminary definitions and basic results. In particular, we recall the definition of the modules $D\left(\xi_{1}, \ldots, \xi_{p}\right)$, the classification of regular minimal affinizations of type $D$, and the definition of graded limits. In Section 3 we state Theorems 3.1 and 3.2, and discuss some of their corollaries. The proofs of Theorems 3.1 and 3.2 is given in Section 4 .

\section{Preliminaries}

\subsection{Simple Lie algebra of type $D_{n}$}

Let $\widehat{I}=\{0,1, \ldots, n\}$ and $\widehat{C}=\left(c_{i j}\right)_{i, j \in \widehat{I}}$ be the Cartan matrix of type $D_{n}^{(1)}$ whose Dynkin diagram is as follows:

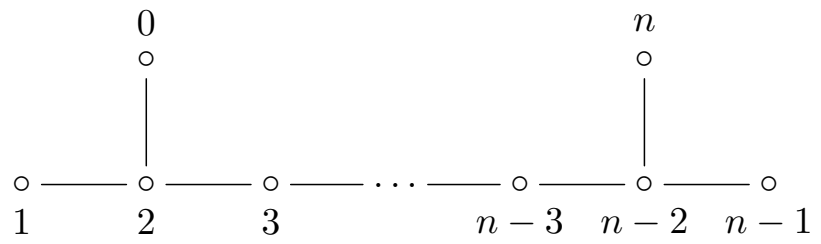


Let $J \subseteq \widehat{I}$ be a subset. In this paper, by abuse of notation, we sometimes denote by $J$ the subdiagram of (2.1) whose vertices are $J$.

Let $I=\widehat{I} \backslash\{0\}, C=\left(c_{i j}\right)_{i, j \in I}$ be the Cartan matrix of type $D_{n}$, and $\mathfrak{g}$ the complex simple Lie algebra associated with $C$. Let $\mathfrak{h}$ be a Cartan subalgebra and $\mathfrak{b}$ a Borel subalgebra containing $\mathfrak{h}$. Denote by $\Delta$ the root system and by $\Delta_{+}$the set of positive roots, and let $\theta \in \Delta_{+}$be the highest root. Let $\alpha_{i}$ and $\varpi_{i}(i \in I)$ be the simple roots and fundamental weights respectively, and set $\varpi_{0}=0$ for convenience. Let $P$ be the weight lattice and $P^{+}$the set of dominant integral weights. Let $W$ denote the Weyl group with simple reflections $s_{i}(i \in I)$, and $w_{\circ} \in W$ the longest element.

For each $\alpha \in \Delta$ denote by $\mathfrak{g}_{\alpha}$ the corresponding root space, and fix nonzero elements $e_{\alpha} \in \mathfrak{g}_{\alpha}$, $f_{\alpha} \in \mathfrak{g}_{-\alpha}$ and $\alpha^{\vee} \in \mathfrak{h}$ such that

$$
\left[e_{\alpha}, f_{\alpha}\right]=\alpha^{\vee}, \quad\left[\alpha^{\vee}, e_{\alpha}\right]=2 e_{\alpha}, \quad\left[\alpha^{\vee}, f_{\alpha}\right]=-2 f_{\alpha} .
$$

We also use the notation $e_{i}=e_{\alpha_{i}}, f_{i}=f_{\alpha_{i}}$ for $i \in I$. Set $\mathfrak{n}_{ \pm}=\bigoplus_{\alpha \in \Delta_{+}} \mathfrak{g}_{ \pm \alpha}$. For a subset $J \subseteq I$, denote by $\mathfrak{g}_{J} \subseteq \mathfrak{g}$ the semisimple Lie subalgebra corresponding to $J$, and let $\mathfrak{h}_{J}=\sum_{i \in J} \mathbb{C} \alpha_{i}^{\vee} \subseteq \mathfrak{h}$.

\subsection{Affine Lie algebra of type $D_{n}^{(1)}$}

Let $\widehat{\mathfrak{g}}=\mathfrak{g} \otimes \mathbb{C}\left[t, t^{-1}\right] \oplus \mathbb{C} K \oplus \mathbb{C} d$ be the affine Lie algebra with Cartan matrix $\widehat{C}$, where $K$ is the canonical central element and $d$ is the degree operator. Naturally $\mathfrak{g}$ is regarded as a Lie subalgebra of $\widehat{\mathfrak{g}}$. Define a Cartan subalgebra $\widehat{\mathfrak{h}}$ and a Borel subalgebra $\widehat{\mathfrak{b}}$ as follows:

$$
\widehat{\mathfrak{h}}=\mathfrak{h} \oplus \mathbb{C} K \oplus \mathbb{C} d, \quad \widehat{\mathfrak{b}}=\widehat{\mathfrak{h}} \oplus \mathfrak{n}_{+} \oplus \mathfrak{g} \otimes t \mathbb{C}[t] .
$$

Set $\widehat{\mathfrak{n}}_{+}=\mathfrak{n}_{+} \oplus \mathfrak{g} \otimes t \mathbb{C}[t]$. We often consider $\mathfrak{h}^{*}$ as a subspace of $\widehat{\mathfrak{h}}^{*}$ by setting $\langle K, \lambda\rangle=\langle d, \lambda\rangle=0$ for $\lambda \in \mathfrak{h}^{*}$. Let $\widehat{\Delta}$ be the root system of $\widehat{\mathfrak{g}}, \widehat{\Delta}_{+}$the set of positive roots, $\widehat{\Delta}^{\text {re }}$ the set of real roots and $\widehat{\Delta}_{+}^{\mathrm{re}}=\widehat{\Delta}_{+} \cap \widehat{\Delta}^{\mathrm{re}}$. Set $\alpha_{0}=\delta-\theta, e_{0}=f_{\theta} \otimes t, f_{0}=e_{\theta} \otimes t^{-1}$ and $\alpha_{0}^{\vee}=K-\theta^{\vee}$.

Denote by $\Lambda_{0} \in \widehat{\mathfrak{h}}^{*}$ the unique element satisfying $\left\langle K, \Lambda_{0}\right\rangle=1$ and $\left\langle\mathfrak{h}, \Lambda_{0}\right\rangle=\left\langle d, \Lambda_{0}\right\rangle=0$, and define $\widehat{P}, \widehat{P}^{+} \subseteq \widehat{\mathfrak{h}}^{*}$ by

$$
\widehat{P}=P \oplus \mathbb{Z} \Lambda_{0} \oplus \mathbb{C} \delta \quad \text { and } \quad \widehat{P}^{+}=\left\{\xi \in \widehat{P} \mid\left\langle\alpha_{i}^{\vee}, \xi\right\rangle \geq 0 \text { for all } i \in \widehat{I}\right\} .
$$

Let $\widehat{W}$ denote the Weyl group of $\widehat{\mathfrak{g}}$ with simple reflections $s_{i}(i \in \widehat{I})$. We regard $W$ naturally as a subgroup of $\widehat{W}$. Let $\ell: \widehat{W} \rightarrow \mathbb{Z}_{\geq 0}$ be the length function. Let $($, ) be the unique non-degenerate $\widehat{W}$-invariant symmetric bilinear form on $\widehat{\mathfrak{h}}^{*}$ satisfying

$$
(\alpha, \alpha)=2 \quad \text { for } \quad \alpha \in \widehat{\Delta}^{\mathrm{re}}, \quad\left(\mathfrak{h}^{*}, \delta\right)=\left(\mathfrak{h}^{*}, \Lambda_{0}\right)=\left(\Lambda_{0}, \Lambda_{0}\right)=0 \quad \text { and } \quad\left(\delta, \Lambda_{0}\right)=1 .
$$

Let $\Sigma$ be the group of Dynkin diagram automorphisms of $\widehat{\mathfrak{g}}$, which naturally acts on $\widehat{\mathfrak{h}}^{*}$ and $\widehat{\mathfrak{g}}$, and $\widetilde{W}$ the subgroup of GL( $\left(\widehat{\mathfrak{h}}^{*}\right)$ generated by $\widehat{W}$ and $\Sigma$. Note that we have $\widetilde{W}=\Sigma \ltimes \widehat{W}$. The length function $\ell$ is extended on $\widetilde{W}$ by setting $\ell(\tau w)=\ell(w)$ for $\tau \in \Sigma, w \in \widehat{W}$.

Denote by $V(\lambda)$ for $\lambda \in P^{+}$the simple $\mathfrak{g}$-module with highest weight $\lambda$, and by $\widehat{V}(\Lambda)$ for $\Lambda \in \widehat{P}^{+}$the simple highest weight $\widehat{\mathfrak{g}}$-module with highest weight $\Lambda$. For a finite-dimensional semisimple $\mathfrak{h}$-module (resp. $\widehat{\mathfrak{h}}$-module) $M$ we denote by $\operatorname{ch}_{\mathfrak{h}} M \in \mathbb{Z}\left[\mathfrak{h}^{*}\right]\left(\right.$ resp. $\left.\operatorname{ch}_{\widehat{\mathfrak{h}}} M \in \mathbb{Z}\left[\widehat{\mathfrak{h}}^{*}\right]\right)$ its character with respect to $\mathfrak{h}$ (resp. $\widehat{\mathfrak{h}}$ ). We will omit the subscript $\mathfrak{h}$ or $\widehat{\mathfrak{h}}$ when it is obvious from the context. 


\subsection{Loop algebras and current algebras}

Given a Lie algebra $\mathfrak{a}$, its loop algebra $\mathbf{L a}$ is defined as the tensor product $\mathfrak{a} \otimes \mathbb{C}\left[t, t^{-1}\right]$ with the Lie algebra structure given by $[x \otimes f, y \otimes g]=[x, y] \otimes f g$. Let $\mathfrak{a}[t]$ and $t^{k} \mathfrak{a}[t]$ for $k \in \mathbb{Z}_{>0}$ denote the Lie subalgebras $\mathfrak{a} \otimes \mathbb{C}[t]$ and $\mathfrak{a} \otimes t^{k} \mathbb{C}[t]$ respectively. The Lie algebra $\mathfrak{a}[t]$ is called the current algebra associated with $\mathfrak{a}$.

For $a \in \mathbb{C}^{\times}$, let $\mathrm{ev}_{a}: \mathbf{L} \mathfrak{g} \rightarrow \mathfrak{g}$ denote the evaluation map defined by $\mathrm{ev}_{a}(x \otimes f)=f(a) x$, and let $V(\lambda, a)$ for $\lambda \in P^{+}$be the evaluation module which is the simple $\mathbf{L} \mathfrak{g}$-module defined by the pull-back of $V(\lambda)$ with respect to $\mathrm{ev}_{a}$. An evaluation module for $\mathfrak{g}[t]$ is defined similarly and is denoted by $V(\lambda, a)\left(\lambda \in P^{+}, a \in \mathbb{C}\right)$.

\section{$2.4 \widehat{\mathfrak{b}}$-submodules $D\left(\xi_{1}, \ldots, \xi_{p}\right)$}

Let $\xi_{1}, \ldots, \xi_{p}$ be a sequence of elements belonging to the Weyl group orbits $\widehat{W}\left(\widehat{P}^{+}\right)$of dominant integral weights of $\widehat{\mathfrak{g}}$. We define a $\widehat{\mathfrak{b}}$-module $D\left(\xi_{1}, \ldots, \xi_{p}\right)$ as follows. For each $1 \leq j \leq p$ let $\Lambda^{j} \in \widehat{P}^{+}$be the unique element satisfying $\xi_{j} \in \widehat{W} \Lambda^{j}$, and take a nonzero vector $v_{\xi_{j}}$ in the 1-dimensional weight space $\widehat{V}\left(\Lambda^{j}\right)_{\xi_{j}}$. Then define

$$
D\left(\xi_{1}, \ldots, \xi_{p}\right)=U(\widehat{\mathfrak{b}})\left(v_{\xi_{1}} \otimes \cdots \otimes v_{\xi_{p}}\right) \subseteq \widehat{V}\left(\Lambda^{1}\right) \otimes \cdots \otimes \widehat{V}\left(\Lambda^{p}\right) .
$$

If $\left(\alpha_{i}, \xi_{j}\right) \leq 0$ for all $i \in I$ and $1 \leq j \leq p$, then $D\left(\xi_{1}, \ldots, \xi_{p}\right)$ can be regarded as a $\mathfrak{g}[t] \oplus \mathbb{C} K \oplus \mathbb{C} d$ module and in particular a $\mathfrak{g}[t]$-module.

Some of $D\left(\xi_{1}, \ldots, \xi_{p}\right)$ are realized in a different way. To introduce this, we prepare some notation. Assume that $V$ is a $\widehat{\mathfrak{g}}$-module and $D$ is a $\widehat{\mathfrak{b}}$-submodule of $V$. For $\tau \in \Sigma$, we denote by $F_{\tau} V$ the pull-back $\left(\tau^{-1}\right)^{*} V$ with respect to the Lie algebra automorphism $\tau^{-1}$ on $\widehat{\mathfrak{g}}$, and define a $\widehat{\mathfrak{b}}$-submodule $F_{\tau} D \subseteq F_{\tau} V$ in the obvious way. It is easily proved that

$$
F_{\tau} D\left(\xi_{1}, \ldots, \xi_{p}\right) \cong D\left(\tau \xi_{1}, \ldots, \tau \xi_{p}\right) .
$$

For $i \in \widehat{I}$ let $\widehat{\mathfrak{p}}_{i}$ denote the parabolic subalgebra $\widehat{\mathfrak{b}} \oplus \mathbb{C} f_{i} \subseteq \widehat{\mathfrak{g}}$, and set $F_{i} D=U\left(\widehat{\mathfrak{p}}_{i}\right) D \subseteq V$ to be the $\widehat{\mathfrak{p}}_{i}$-submodule generated by $D$. Finally we define $F_{w} D$ for $w \in \widetilde{W}$ as follows: let $\tau \in \Sigma$ and $w^{\prime} \in \widehat{W}$ be the elements such that $w=\tau w^{\prime}$, and choose a reduced expression $w^{\prime}=s_{i_{1}} \cdots s_{i_{k}}$. Then we set

$$
F_{w} D=F_{\tau} F_{i_{1}} \cdots F_{i_{k}} D \subseteq F_{\tau} V .
$$

Proposition 2.1 ([21, Proposition 2.7]). Let $\Lambda^{1}, \ldots, \Lambda^{p}$ be a sequence of elements of $\widehat{P}^{+}$, and $w_{1}, \ldots, w_{p}$ a sequence of elements of $\widetilde{W}$. We write $w_{[r, s]}=w_{r} w_{r+1} \cdots w_{s}$ for $r \leq s$, and assume that $\ell\left(w_{[1, p]}\right)=\sum_{j=1}^{p} \ell\left(w_{j}\right)$. Then we have

$$
\begin{aligned}
& D\left(w_{[1,1]} \Lambda^{1}, w_{[1,2]} \Lambda^{2}, \ldots, w_{[1, p-1]} \Lambda^{p-1}, w_{[1, p]} \Lambda^{p}\right) \\
& \quad \cong F_{w_{1}}\left(D\left(\Lambda^{1}\right) \otimes F_{w_{2}}\left(D\left(\Lambda^{2}\right) \otimes \cdots \otimes F_{w_{p-1}}\left(D\left(\Lambda^{p-1}\right) \otimes F_{w_{p}} D\left(\Lambda^{p}\right)\right) \cdots\right)\right) .
\end{aligned}
$$

Let $\mathcal{D}_{i}$ for $i \in \widehat{I}$ be a linear operator on $\mathbb{Z}[\widehat{P}]$ defined by

$$
\mathcal{D}_{i}(f)=\frac{f-e^{-\alpha_{i}} s_{i}(f)}{1-e^{-\alpha_{i}}},
$$

where $e^{\lambda}(\lambda \in \widehat{P})$ are the generators of $\mathbb{Z}[\widehat{P}]$. For $w \in \widehat{W}$ with a reduced expression $w=s_{i_{1}} \cdots s_{i_{k}}$, we set $\mathcal{D}_{w}=\mathcal{D}_{i_{1}} \cdots \mathcal{D}_{i_{k}}$. If $w \in \widetilde{W}$ and $w=\tau w^{\prime}\left(\tau \in \Sigma, w^{\prime} \in \widehat{W}\right)$, we set $\mathcal{D}_{w}=\tau \mathcal{D}_{w^{\prime}}$. The operator $\mathcal{D}_{w}$ is called a Demazure operator. The character of the right-hand side of (2.2) is expressed using Demazure operators by [16, Theorem 5], and as a consequence we have the following (see also [21, Corollary 2.8]). 
Proposition 2.2. Let $\Lambda^{j} \in \widehat{P}^{+}$and $w_{j} \in \widetilde{W}(1 \leq j \leq p)$ be as in Proposition 2.1. Then we have

$$
\begin{aligned}
\operatorname{ch}_{\widehat{\mathfrak{h}}} & D\left(w_{[1,1]} \Lambda^{1}, w_{[1,2]} \Lambda^{2}, \ldots, w_{[1, p-1]} \Lambda^{p-1}, w_{[1, p]} \Lambda^{p}\right) \\
& =\mathcal{D}_{w_{1}}\left(e^{\Lambda^{1}} \cdot \mathcal{D}_{w_{2}}\left(e^{\Lambda^{2}} \cdots \mathcal{D}_{w_{p-1}}\left(e^{\Lambda^{p-1}} \cdot \mathcal{D}_{w_{p}}\left(e^{\Lambda^{p}}\right)\right) \cdots\right)\right) .
\end{aligned}
$$

\subsection{Quantum loop algebras and their representations}

The quantum loop algebra $U_{q}(\mathbf{L} \mathfrak{g})$ is a $\mathbb{C}(q)$-algebra generated by $x_{i, r}^{ \pm}, k_{i}^{ \pm 1}$ and $h_{i, m}(i \in I$, $r \in \mathbb{Z}, m \in \mathbb{Z} \backslash\{0\}$ ) subject to certain relations (see, e.g., [6, Section 12.2]). $U_{q}(\mathbf{L g})$ has a Hopf algebra structure $[6,17]$. In particular if $V$ and $W$ are $U_{q}(\mathbf{L g})$-modules then $V \otimes W$ and $V^{*}$ are also $U_{q}(\mathbf{L g})$-modules, and we have $(V \otimes W)^{*} \cong W^{*} \otimes V^{*}$.

Denote by $U_{q}\left(\mathbf{L n}_{ \pm}\right)$and $U_{q}(\mathbf{L} \mathfrak{h})$ the subalgebras of $U_{q}(\mathbf{L g})$ generated by $\left\{x_{i, r}^{ \pm} \mid i \in I, r \in \mathbb{Z}\right\}$ and $\left\{k_{i}^{ \pm 1}, h_{i, m} \mid i \in I, m \in \mathbb{Z} \backslash\{0\}\right\}$ respectively. Denote by $U_{q}(\mathfrak{g})$ the subalgebra generated by $\left\{x_{i, 0}^{ \pm}, k_{i}^{ \pm 1} \mid i \in I\right\}$, which is isomorphic to the quantized enveloping algebra associated with g. For a subset $J \subseteq I$, let $U_{q}\left(\mathbf{L g}_{J}\right)$ denote the subalgebra generated by $\left\{x_{i, r}^{ \pm}, k_{i}^{ \pm 1}, h_{i, m} \mid i \in J\right.$, $r \in \mathbb{Z}, m \in \mathbb{Z} \backslash\{0\}\}$.

We recall basic results on finite-dimensional $U_{q}(\mathfrak{g})$ - and $U_{q}(\mathbf{L} \mathfrak{g})$-modules. Note that in the present paper we assume that $\mathfrak{g}$ is of type $D$, and when $\mathfrak{g}$ is non-simply laced some of indeterminates $q$ appearing below should be replaced by $q_{i}=q^{d_{i}}$ with suitable $d_{i} \in \mathbb{Z}_{>0}$.

A $U_{q}(\mathfrak{g})$-module (or $U_{q}(\mathbf{L} \mathfrak{g})$-module) $V$ is said to be of type 1 if $V$ satisfies

$$
V=\bigoplus_{\lambda \in P} V_{\lambda}, \quad V_{\lambda}=\left\{v \in V \mid k_{i} v=q^{\left\langle\alpha_{i}^{\vee}, \lambda\right\rangle} v\right\} .
$$

In this article we will only consider modules of type 1 . For a finite-dimensional module $V$ of type 1 , we set $\operatorname{ch} V=\sum_{\lambda \in P} e^{\lambda} \operatorname{dim} V_{\lambda} \in \mathbb{Z}[P]$. For $\lambda \in P^{+}$we denote by $V_{q}(\lambda)$ the finitedimensional simple $U_{q}(\mathfrak{g})$-module of type 1 with highest weight $\lambda$. The category of finitedimensional $U_{q}(\mathfrak{g})$-modules of type 1 is semisimple, and every simple object is isomorphic to $V_{q}(\lambda)$ for some $\lambda \in P^{+}$.

We say that a $U_{q}(\mathbf{L g})$-module $V$ is highest $\ell$-weight with highest $\ell$-weight vector $v$ and highest

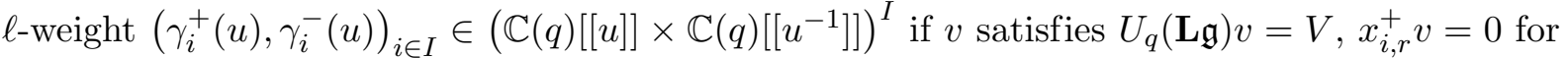
all $i \in I, r \in \mathbb{Z}$, and $\phi_{i}^{ \pm}(u) v=\gamma_{i}^{ \pm}(u) v$ for all $i \in I$. Here $\phi_{i}^{ \pm}(u) \in U_{q}(\mathbf{L h})\left[\left[u^{ \pm 1}\right]\right]$ are defined as follows:

$$
\phi_{i}^{ \pm}(u)=k_{i}^{ \pm 1} \exp \left( \pm\left(q-q^{-1}\right) \sum_{r=1}^{\infty} h_{i, \pm r} u^{ \pm r}\right) .
$$

Theorem 2.3 ([8]).

(i) If $V$ is a finite-dimensional simple $U_{q}(\mathbf{L g})$-module of type 1 , then $V$ is highest $\ell$-weight, and its highest $\ell$-weight $\left(\gamma_{i}^{+}(u), \gamma_{i}^{-}(u)\right)_{i \in I}$ satisfies

$$
\gamma_{i}^{ \pm}(u)=q^{\operatorname{deg} \boldsymbol{\pi}_{i}(u)}\left(\frac{\boldsymbol{\pi}_{i}\left(q^{-1} u\right)}{\boldsymbol{\pi}_{i}(q u)}\right)^{ \pm}
$$

for some polynomials $\boldsymbol{\pi}_{i}(u) \in \mathbb{C}(q)[u]$ whose constant terms are 1 . Here ()$^{ \pm}$denote the expansions at $u=0$ and $u=\infty$ respectively.

(ii) Conversely, for every I-tuple of polynomials $\boldsymbol{\pi}=\left(\boldsymbol{\pi}_{1}(u), \ldots, \boldsymbol{\pi}_{n}(u)\right)$ such that $\boldsymbol{\pi}_{i}(0)=1$, there exists a unique (up to isomorphism) finite-dimensional simple highest $\ell$-weight $U_{q}(\mathbf{L} \mathfrak{g})$-module of type 1 with highest $\ell$-weight $\left(\gamma_{i}^{+}(u), \gamma_{i}^{-}(u)\right)_{i \in I}$ satisfying $(2.3)$. 
The $I$-tuple of polynomials $\boldsymbol{\pi}=\left(\boldsymbol{\pi}_{1}(u), \ldots, \boldsymbol{\pi}_{n}(u)\right)$ are called Drinfeld polynomials, and we will say by abuse of terminology that the highest $\ell$-weight of $V$ is $\boldsymbol{\pi}$ if the highest $\ell$-weight $\left(\gamma_{i}^{+}(u), \gamma_{i}^{-}(u)\right)_{i \in I}$ of $V$ satisfies $(2.3)$. We denote by $L_{q}(\boldsymbol{\pi})$ the finite-dimensional simple $U_{q}(\mathbf{L} \mathfrak{g})$ module of type 1 with highest $\ell$-weight $\boldsymbol{\pi}$, and by $v_{\boldsymbol{\pi}}$ a highest $\ell$-weight vector of $L_{q}(\boldsymbol{\pi})$.

Let $i \mapsto \bar{i}$ be the bijection $I \rightarrow I$ determined by $\alpha_{\bar{i}}=-w_{\circ}\left(\alpha_{i}\right)$.

Lemma 2.4 ([6]). For any Drinfeld polynomials $\boldsymbol{\pi}$ we have

$$
L_{q}(\boldsymbol{\pi})^{*} \cong L_{q}\left(\boldsymbol{\pi}^{*}\right)
$$

as $U_{q}(\mathbf{L} \mathfrak{g})$-modules, where $\boldsymbol{\pi}^{*}=\left(\boldsymbol{\pi}_{\bar{i}}\left(q^{-h^{\vee}} u\right)\right)_{i \in I}$ and $h^{\vee}$ is the dual Coxeter number.

\subsection{Minimal affinizations}

For an $I$-tuple of polynomials $\boldsymbol{\pi}=\left(\boldsymbol{\pi}_{i}(u)\right)_{i \in I}$, set $\operatorname{wt}(\boldsymbol{\pi})=\sum_{i \in I} \varpi_{i} \operatorname{deg} \boldsymbol{\pi}_{i} \in P^{+}$.

Definition 2.5 ([1]). Let $\lambda \in P^{+}$.

(i) A simple finite-dimensional $U_{q}(\mathbf{L} \mathfrak{g})$-module $L_{q}(\boldsymbol{\pi})$ is said to be an affinization of $V_{q}(\lambda)$ if $\operatorname{wt}(\boldsymbol{\pi})=\lambda$.

(ii) Affinizations $V$ and $W$ of $V_{q}(\lambda)$ are said to be equivalent if they are isomorphic as $U_{q}(\mathfrak{g})$ modules. We denote by $[V]$ the equivalence class of $V$.

If $V$ is an affinization of $V_{q}(\lambda)$, as a $U_{q}(\mathfrak{g})$-module we have

$$
V \cong V_{q}(\lambda) \oplus \bigoplus_{\mu<\lambda} V_{q}(\mu)^{\oplus m_{\mu}(V)}
$$

with some $m_{\mu}(V) \in \mathbb{Z}_{\geq 0}$. Let $V$ and $W$ be affinizations of $V_{q}(\lambda)$, and define $m_{\mu}(V), m_{\mu}(W)$ as above. We write $[V] \leq[W]$ if for all $\mu \in P^{+}$, either of the following holds:

(i) $m_{\mu}(V) \leq m_{\mu}(W)$, or

(ii) there exists some $\nu>\mu$ such that $m_{\nu}(V)<m_{\nu}(W)$.

Then $\leq$ defines a partial ordering on the set of equivalence classes of affinizations of $V_{q}(\lambda)[1$, Proposition 3.7].

Definition 2.6 ([1]). We say an affinization $V$ of $V_{q}(\lambda)$ is minimal if $[V]$ is minimal in the set of equivalence classes of affinizations of $V_{q}(\lambda)$ with respect to this ordering.

For $i \in I, a \in \mathbb{C}(q)^{\times}$and $m \in \mathbb{Z}_{>0}$, define an $I$-tuple of polynomials $\boldsymbol{\pi}_{m, a}^{(i)}$ by

$$
\left(\boldsymbol{\pi}_{m, a}^{(i)}\right)_{j}(u)= \begin{cases}\left(1-a q^{-m+1} u\right)\left(1-a q^{-m+3} u\right) \cdots\left(1-a q^{m-1} u\right), & j=i \\ 1, & j \neq i\end{cases}
$$

We set $\boldsymbol{\pi}_{0, a}^{(i)}=(1,1, \ldots, 1)$ for every $i \in I$ and $a \in \mathbb{C}(q)^{\times}$. The simple modules $L_{q}\left(\boldsymbol{\pi}_{m, a}^{(i)}\right)$ are called Kirillov-Reshetikhin modules.

Let us recall the classification of minimal affinizations in the regular case of type $D$, which was given in [10]. (Similar results also hold in type $E$. See [7] for type $A B C F G$, in which minimal affinizations are unique up to equivalence.) For that, we fix several notation. Set $S=\{1, n-1, n\} \subseteq I$ and define the subsets $I_{s} \subseteq I(s \in S)$ by $I_{1}=\{1,2, \ldots, n-3\}, I_{n-1}=$ $\{n-1\}, I_{n}=\{n\}$. Note that $I_{s}$ is the connected component of the subdiagram $I \backslash\{n-2\}$ containing $s$, and $I \backslash I_{s}$ is the maximal type $A$ subdiagram of $I$ not containing $s$. For $s \in S$, $\varepsilon \in\{ \pm\}, \lambda=\sum_{i \in I} \lambda_{i} \varpi_{i} \in P^{+}$and $a \in \mathbb{C}(q)^{\times}$, define Drinfeld polynomials $\boldsymbol{\pi}_{s}^{\varepsilon}(\lambda, a)$ as follows: 
○ When $s=1$, set $\boldsymbol{\pi}_{1}^{\varepsilon}(\lambda, a)=\prod_{i \in I} \boldsymbol{\pi}_{\lambda_{i}, a_{i}}^{(i)}$ (the product being defined component-wise) with $a_{1}=a$ and

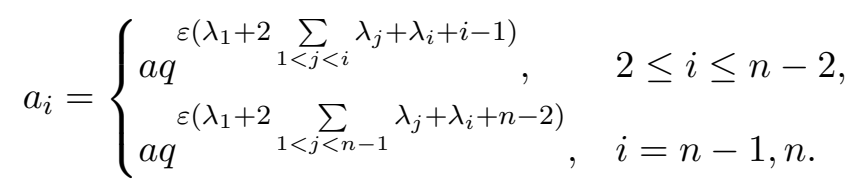

○ When $s=n-1$ or $n$, set $\boldsymbol{\pi}_{s}^{\varepsilon}(\lambda, a)=\prod_{i \in I} \boldsymbol{\pi}_{\lambda_{i}, a_{i}}^{(i)}$ with $a_{1}=a$ and

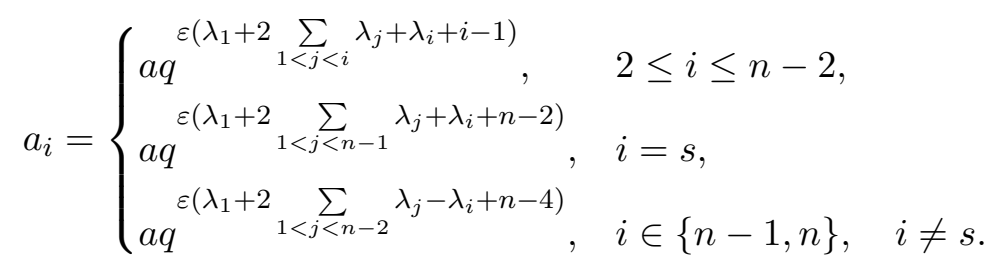

Remark 2.7. The Drinfeld polynomials $\boldsymbol{\pi}_{s}^{\varepsilon}(\lambda, a)$ are determined so that they satisfy the following property: if $r \in S \backslash\{s\}$ and $J=I \backslash I_{r}$, the simple $U_{q}\left(\mathbf{L g}_{J}\right)$-module $L_{q}\left(\boldsymbol{\pi}_{s}^{\varepsilon}(\lambda, a)_{J}\right)$ is a minimal affinization of the $U_{q}\left(\mathfrak{g}_{J}\right)$-module $V_{q}\left(\left.\lambda\right|_{\mathfrak{h}_{J}}\right)$, where $\boldsymbol{\pi}_{s}^{\varepsilon}(\lambda, a)_{J}$ denotes the $J$-tuple $\left(\boldsymbol{\pi}_{s}^{\varepsilon}(\lambda, a)_{j}(u)\right)_{j \in J}($ see $[10$, Theorem 3.1]).

Define the support of $\lambda=\sum_{i} \lambda_{i} \varpi_{i} \in P^{+}$by

$$
\operatorname{supp}(\lambda)=\left\{i \in I \mid \lambda_{i}>0\right\} \subseteq I
$$

Theorem 2.8 ([10, Theorem 6.1]). Let $\lambda \in P^{+}$.

(i) If $\operatorname{supp}(\lambda) \cap I_{s}=\varnothing$ for some $s \in S$, then there exists a unique equivalence class of minimal affinizations of $V_{q}(\lambda)$, and the equivalence class is given by

$$
\left\{L_{q}\left(\boldsymbol{\pi}_{r}^{\varepsilon}(\lambda, a)\right) \mid \varepsilon \in\{ \pm\}, a \in \mathbb{C}(q)^{\times}\right\}
$$

with $r \in S \backslash\{s\}$ (here the choice of $r$ is irrelevant since $\boldsymbol{\pi}_{r}^{ \pm}(\lambda, a)=\boldsymbol{\pi}_{r^{\prime}}^{ \pm}(\lambda, a)$ holds for $r$, $\left.r^{\prime} \in S \backslash\{s\}\right)$.

(ii) If $\operatorname{supp}(\lambda) \cap I_{s} \neq \varnothing$ for all $s \in S$ and $\lambda_{n-2}>0$, then there exist exactly three equivalence classes of minimal affinizations of $V_{q}(\lambda)$, and for each $s \in S$

$$
\left\{L_{q}\left(\boldsymbol{\pi}_{s}^{\varepsilon}(\lambda, a)\right) \mid \varepsilon \in\{ \pm\}, a \in \mathbb{C}(q)^{\times}\right\}
$$

forms an equivalence class.

We call $\lambda \in P^{+}$regular if $\lambda$ satisfies one of the assumptions of (i) or (ii) in Theorem 2.8. We call a minimal affinization is regular if its highest weight is regular.

Remark 2.9 ([9]). In the remaining case when $\operatorname{supp}(\lambda) \cap I_{s} \neq \varnothing$ for all $s \in S$ and $\lambda_{n-2}=0$, the number of equivalence classes of minimal affinizations increases unboundedly with $\lambda$, and the classification of minimal affinizations has not been given except for the type $D_{4}$. 


\subsection{Classical limits and graded limits}

Let $\mathbf{A}=\mathbb{C}\left[q, q^{-1}\right]$ be the ring of Laurent polynomials with complex coefficients, and denote by $U_{\mathbf{A}}(\mathbf{L} \mathfrak{g})$ the $\mathbf{A}$-subalgebra of $U_{q}(\mathbf{L} \mathfrak{g})$ generated by $\left\{k_{i}^{ \pm 1},\left(x_{i, r}^{ \pm}\right)^{k} /[k]_{q} ! \mid i \in I, r \in \mathbb{Z}, k \in \mathbb{Z}_{>0}\right\}$, where we set $[k]_{q}=\left(q^{k}-q^{-k}\right) /\left(q-q^{-1}\right)$ and $[k]_{q} !=[k]_{q}[k-1]_{q} \cdots[1]_{q}$. Define $U_{\mathbf{A}}(\mathfrak{g}) \subseteq U_{q}(\mathfrak{g})$ in a similar way. We define $\mathbb{C}$-algebras $U_{1}(\mathbf{L} \mathfrak{g})$ and $U_{1}(\mathfrak{g})$ by

$$
U_{1}(\mathbf{L} \mathfrak{g})=\mathbb{C} \otimes_{\mathbf{A}} U_{\mathbf{A}}(\mathbf{L} \mathfrak{g}) \quad \text { and } \quad U_{1}(\mathfrak{g})=\mathbb{C} \otimes_{\mathbf{A}} U_{\mathbf{A}}(\mathfrak{g}),
$$

where we identify $\mathbb{C}$ with $\mathbf{A} /\langle q-1\rangle$. Then the following $\mathbb{C}$-algebra isomorphisms are known to hold [17], [6, Proposition 9.3.10]:

$$
U(\mathbf{L} \mathfrak{g}) \cong U_{1}(\mathbf{L} \mathfrak{g}) /\left\langle k_{i}-1 \mid i \in I\right\rangle_{U_{1}(\mathbf{L} \mathfrak{g})}, \quad U(\mathfrak{g}) \cong U_{1}(\mathfrak{g}) /\left\langle k_{i}-1 \mid i \in I\right\rangle_{U_{1}(\mathfrak{g})},
$$

where $\left\langle k_{i}-1 \mid i \in I\right\rangle_{U_{1}(\mathbf{L g})}$ denotes the two-sided ideal of $U_{1}(\mathbf{L} \mathfrak{g})$ generated by $\left\{k_{i}-1 \mid i \in I\right\}$, and $\left\langle k_{i}-1 \mid i \in I\right\rangle_{U_{1}(\mathfrak{g})}$ is defined similarly.

Let $\boldsymbol{\pi}=\left(\boldsymbol{\pi}_{1}(u), \ldots, \boldsymbol{\pi}_{n}(u)\right)$ be Drinfeld polynomials, and assume that there exists $b \in \mathbb{C}^{\times}$ such that all the roots of $\boldsymbol{\pi}_{i}(u)$ 's are contained in the set $b q^{\mathbb{Z}}$ (it is known that in order to describe the category of finite-dimensional $U_{q}(\mathbf{L} \mathfrak{g})$-modules, it is essentially enough to consider representations attached to such families of Drinfeld polynomials. For example, see [13, Section 3.7]). Note that $\boldsymbol{\pi}_{s}^{ \pm}(\lambda, a)$ satisfies this assumptions when $a \in \mathbb{C}^{\times} q^{\mathbb{Z}}$. Let $L_{\mathbf{A}}(\boldsymbol{\pi})$ be the $U_{\mathbf{A}}(\mathbf{L} \mathfrak{g})$-submodule of $L_{q}(\boldsymbol{\pi})$ generated by a highest $\ell$-weight vector $v_{\boldsymbol{\pi}}$. Then by the isomorphism (2.4),

$$
\overline{L_{q}(\boldsymbol{\pi})}=\mathbb{C} \otimes_{\mathbf{A}} L_{\mathbf{A}}(\boldsymbol{\pi})
$$

becomes a finite-dimensional $\mathbf{L} \mathfrak{g}$-module, which is called the classical limit of $L_{q}(\boldsymbol{\pi})$.

Define a Lie algebra automorphism $\varphi_{b}: \mathfrak{g}[t] \rightarrow \mathfrak{g}[t]$ by

$$
\varphi_{b}(x \otimes f(t))=x \otimes f(t-b) \quad \text { for } \quad x \in \mathfrak{g}, f \in \mathbb{C}[t] .
$$

We consider $\overline{L_{q}(\boldsymbol{\pi})}$ as a $\mathfrak{g}[t]$-module by restriction, and define a $\mathfrak{g}[t]$-module $L(\boldsymbol{\pi})$ by the pullback $\varphi_{b}^{*}\left(\overline{L_{q}(\boldsymbol{\pi})}\right)$. We call $L(\boldsymbol{\pi})$ the graded limit of $L_{q}(\boldsymbol{\pi})$. In fact, at least when $\boldsymbol{\pi}=\boldsymbol{\pi}_{s}^{ \pm}(\lambda, a)$, it turns out later from our main theorems that $L(\boldsymbol{\pi})$ is a graded $\mathfrak{g}[t]$-module, which justifies the name "graded limit". Set $\bar{v}_{\boldsymbol{\pi}}=1 \otimes_{\mathbf{A}} v_{\boldsymbol{\pi}} \in L(\boldsymbol{\pi})$, which generates $L(\boldsymbol{\pi})$ as a $\mathfrak{g}[t]$-module. The following properties of graded limits are easily proved from the construction (see [2]).

Lemma 2.10. Assume $\operatorname{wt}(\boldsymbol{\pi})=\lambda$.

(i) There exists a surjective $\mathfrak{g}[t]$-module homomorphism from $L(\boldsymbol{\pi})$ to $V(\lambda, 0)$ mapping $\bar{v}_{\boldsymbol{\pi}}$ to a highest weight vector.

(ii) The vector $\bar{v}_{\boldsymbol{\pi}}$ satisfies the relations

$$
\begin{aligned}
& \mathfrak{n}_{+}[t] \bar{v}_{\boldsymbol{\pi}}=0, \quad\left(h \otimes t^{k}\right) \bar{v}_{\boldsymbol{\pi}}=\delta_{k 0}\langle h, \lambda\rangle \bar{v}_{\boldsymbol{\pi}} \quad \text { for } \quad h \in \mathfrak{h}, k \geq 0, \quad \text { and } \\
& f_{i}^{\left\langle\alpha_{i}^{\vee}, \lambda\right\rangle+1} \bar{v}_{\boldsymbol{\pi}}=0 \quad \text { for } \quad i \in I .
\end{aligned}
$$

(iii) We have

$$
\operatorname{ch} L_{q}(\boldsymbol{\pi})=\operatorname{ch} L(\boldsymbol{\pi}) .
$$

(iv) For every $\mu \in P^{+}$we have

$$
\left[L_{q}(\boldsymbol{\pi}): V_{q}(\mu)\right]=[L(\boldsymbol{\pi}): V(\mu)]
$$

where the left- and right-hand sides are the multiplicities as a $U_{q}(\mathfrak{g})$-module and $\mathfrak{g}$-module, respectively. 


\section{Main theorems and corollaries}

Throughout this section, we fix $\lambda=\sum_{i \in I} \lambda_{i} \varpi_{i} \in P^{+}, \varepsilon \in\{ \pm\}$ and $a \in \mathbb{C}^{\times} q^{\mathbb{Z}}$, and abbreviate $\boldsymbol{\pi}_{s}=\boldsymbol{\pi}_{s}^{\varepsilon}(\lambda, a)$ for $s \in S=\{1, n-1, n\}$.

\subsection{Main theorems}

Denote by $\tau_{0,1} \in \Sigma$ (resp. $\tau_{n-1, n} \in \Sigma$ ) the diagram automorphism interchanging the nodes 0 and 1 (resp. $n-1$ and $n$ ). We will not use other elements of $\Sigma$ in the sequel.

For $s \in S$ and $1 \leq j \leq n$, define $\xi_{j}^{(s)}=\xi_{j}^{(s)}(\lambda) \in \widehat{P}$ as follows:

○ When $s=1$, let $m, m^{\prime}$ be such that $\left\{m, m^{\prime}\right\}=\{n-1, n\}, \lambda_{m}=\max \left\{\lambda_{n-1}, \lambda_{n}\right\}$ and $\lambda_{m^{\prime}}=$ $\min \left\{\lambda_{n-1}, \lambda_{n}\right\}$, and define

$$
\xi_{j}^{(1)}= \begin{cases}\lambda_{j}\left(\varpi_{j}+\Lambda_{0}\right), & 1 \leq j \leq n-2, \\ \lambda_{m^{\prime}}\left(\varpi_{n-1}+\varpi_{n}+\Lambda_{0}\right), & j=n-1, \\ \left(\lambda_{m}-\lambda_{m^{\prime}}\right)\left(\varpi_{m}+\Lambda_{0}\right), & j=n .\end{cases}
$$

$\circ$ When $s=n$, set

$$
\ell= \begin{cases}0, & \text { if } \sum_{i=1}^{n-3} \lambda_{i}<\lambda_{n-1}, \\ \max \left\{1 \leq j \leq n-3 \mid \sum_{i=j}^{n-3} \lambda_{i} \geq \lambda_{n-1}\right\}, & \text { otherwise }\end{cases}
$$

and $\bar{\lambda}=\lambda_{n-1}-\sum_{i=\ell+1}^{n-3} \lambda_{i}$. Then define

$$
\xi_{j}^{(n)}= \begin{cases}\lambda_{j}\left(\varpi_{j}+\Lambda_{0}\right), & 1 \leq j<\ell, \quad j=n-2, n, \\ \lambda_{\ell}\left(\varpi_{\ell}+\Lambda_{0}\right)+\bar{\lambda} \varpi_{n-1}, & j=\ell, \\ \lambda_{j}\left(\varpi_{j}+\varpi_{n-1}+\Lambda_{0}\right)+\delta_{\ell 0} \delta_{j 1} \bar{\lambda}\left(\varpi_{n-1}+\Lambda_{0}\right), & \ell<j<n-2, \\ 0, & j=n-1 .\end{cases}
$$

○ When $s=n-1$, set $\xi_{j}^{(n-1)}(\lambda)=\tau_{n-1, n}\left(\xi_{j}^{(n)}\left(\tau_{n-1, n} \lambda\right)\right)$.

Note that we have $\lambda \equiv \sum_{1 \leq j \leq n} \xi_{j}^{(s)} \bmod \mathbb{Z} \Lambda_{0}+\mathbb{Q} \delta$ for all $s \in S$.

Theorem 3.1. The graded limit $L\left(\boldsymbol{\pi}_{s}\right)$ is isomorphic to $D\left(w_{\circ} \xi_{1}^{(s)}, \ldots, w_{\circ} \xi_{n}^{(s)}\right)$ as a $\mathfrak{g}[t]$-module.

For $\alpha=\sum_{i \in I} n_{i} \alpha_{i} \in \Delta_{+}$, set $\operatorname{supp}(\alpha)=\left\{i \in I \mid n_{i}>0\right\} \subseteq I$. We define a subset $\Delta_{+}^{(s)} \subseteq \Delta_{+}$for $s \in S$ by

$$
\Delta_{+}^{(s)}=\bigcup_{r \in S \backslash\{s\}}\left\{\alpha \in \Delta_{+} \mid \operatorname{supp}(\alpha) \subseteq I \backslash I_{r}\right\}
$$

Note that if $\alpha \in \Delta_{+}^{(s)}$, then the coefficient of $\alpha_{i}$ in $\alpha$ is 0 or 1 for all $i \in I$. 
Theorem 3.2. The graded limit $L\left(\boldsymbol{\pi}_{s}\right)$ is isomorphic to the cyclic $\mathfrak{g}[t]$-module generated by a nonzero vector $v$ subject to the relations

$$
\begin{aligned}
& \mathfrak{n}_{+}[t] v=0, \quad\left(h \otimes t^{k}\right) v=\delta_{k 0}\langle h, \lambda\rangle v \quad \text { for } \quad h \in \mathfrak{h}, \quad k \geq 0, \\
& f_{i}^{\lambda_{i}+1} v=0 \quad \text { for } \quad i \in I \quad \text { and } \quad\left(f_{\alpha} \otimes t\right) v=0 \quad \text { for } \quad \alpha \in \Delta_{+}^{(s)} .
\end{aligned}
$$

We prove Theorems 3.1 and 3.2 in Section 4.

Remark 3.3. The defining relations of Theorem 3.2 were conjectured in [18, Section 5.11], and proved there for $\mathfrak{g}$ of type $D_{4}$. Let $I_{s}^{\prime}=I_{s} \sqcup\{n-2\}, \lambda_{I_{s}^{\prime}}=\sum_{i \in I_{s}^{\prime}} \lambda_{i} \varpi_{i}$ and $\lambda_{I \backslash I_{s}^{\prime}}=\lambda-\lambda_{I_{s}^{\prime}}$. In loc. cit., the author also conjectured that the graded limit $L\left(\boldsymbol{\pi}_{s}\right)$ is isomorphic to the $\mathfrak{g}[t]$-submodule of

$$
L\left(\boldsymbol{\pi}_{s}^{\varepsilon}\left(\lambda_{I_{s}^{\prime}}, a\right)\right) \otimes L\left(\boldsymbol{\pi}_{s}^{\varepsilon}\left(\lambda_{I \backslash I_{s}^{\prime}}, a\right)\right)
$$

generated by the tensor product of highest weight vectors. This is easily deduced from Theorem 3.1 .

\subsection{Corollaries}

The module $D\left(w_{\circ} \xi_{1}^{(s)}, \ldots, w_{\circ} \xi_{n}^{(s)}\right)$ in Theorem 3.1 has another realization introduced in Section 2.4. Define $\sigma \in \widetilde{W}$ by

$$
\sigma=\tau_{0,1} \tau_{n-1, n} s_{1} s_{2} \cdots s_{n-1}
$$

The proof of the following lemma is straightforward.

\section{Lemma 3.4.}

(i) For $0 \leq j \leq n$, we have

$$
\sigma\left(\varpi_{j}+\Lambda_{0}\right) \equiv\left\{\begin{array}{ll}
\varpi_{j+1}+\Lambda_{0}, & 0 \leq j \leq n-3, \\
\varpi_{n-1}+\varpi_{n}+\Lambda_{0}, & j=n-2, \\
\varpi_{n-1}+\varpi_{1}+\Lambda_{0}, & j=n-1, \\
\varpi_{n-1}+\Lambda_{0}, & j=n,
\end{array} \quad \bmod \mathbb{Q} \delta,\right.
$$

and $\sigma\left(\varpi_{n-1}\right) \equiv \varpi_{n-1} \quad \bmod \mathbb{Q} \delta$.

(ii) We have $\ell\left(w_{\circ} \sigma^{n-1}\right)=\ell\left(w_{\circ}\right)+(n-1) \ell(\sigma)$.

Assume $s \neq n-1$ for a while. For $1 \leq j \leq n-1$ define $\Lambda_{j}^{(s)}=\sigma^{-j} \xi_{j}^{(s)}$, and set $\Lambda_{n}^{(s)}=\xi_{n}^{(s)}$. The following assertions are easily checked using Lemma 3.4(i):

$$
\begin{aligned}
\Lambda_{j}^{(1)} & \equiv\left\{\begin{array}{ll}
\lambda_{j} \Lambda_{0}, & 1 \leq j \leq n-2, \\
\lambda_{m^{\prime}} \Lambda_{0}, & j=n-1,
\end{array} \quad \bmod \mathbb{Q} \delta, \quad\right. \text { and } \\
\Lambda_{j}^{(n)} & \equiv \begin{cases}\lambda_{j} \Lambda_{0}, & 1 \leq j<\ell \quad \text { or } \quad j=n-2, \\
\lambda_{\ell} \Lambda_{0}+\bar{\lambda} \varpi_{n-1}, & j=\ell, \\
\lambda_{j}\left(\varpi_{n-1}+\Lambda_{0}\right)+\delta_{\ell 0} \delta_{j 1} \bar{\lambda}\left(\varpi_{n}+\Lambda_{0}\right), & \ell<j<n-2,\end{cases}
\end{aligned}
$$

In particular, each $\Lambda_{j}^{(s)}$ belongs to $\widehat{P}^{+}$. We obtain the following chain of isomorphisms from Theorem 3.1, Lemma 3.4(ii), and Proposition 2.1:

$$
\begin{aligned}
L\left(\boldsymbol{\pi}_{s}\right) & \cong D\left(w_{\circ} \xi_{1}^{(s)}, \ldots, w_{\circ} \xi_{n}^{(s)}\right) \cong D\left(w_{\circ} \xi_{n}^{(s)}, w_{\circ} \xi_{1}^{(s)}, \ldots, w_{\circ} \xi_{n-1}^{(s)}\right) \\
& \cong F_{w_{\circ}}\left(D\left(\Lambda_{n}^{(s)}\right) \otimes F_{\sigma}\left(D\left(\Lambda_{1}^{(s)}\right) \otimes \cdots \otimes F_{\sigma}\left(D\left(\Lambda_{n-2}^{(s)}\right) \otimes F_{\sigma} D\left(\Lambda_{n-1}^{(s)}\right)\right) \cdots\right)\right),
\end{aligned}
$$

where the second isomorphism obviously holds by definition. Hence by Proposition 2.2 and Lemma 2.10(iii), the following holds. 
Corollary 3.5. If $s \in\{1, n\}$, we have

$$
\operatorname{ch} L_{q}\left(\boldsymbol{\pi}_{s}\right)=\left.\mathcal{D}_{w_{\circ}}\left(e^{\Lambda_{n}^{(s)}} \cdot \mathcal{D}_{\sigma}\left(e^{\Lambda_{1}^{(s)}} \cdots \mathcal{D}_{\sigma}\left(e^{\Lambda_{n-2}^{(s)}} \cdot \mathcal{D}_{\sigma}\left(e^{\Lambda_{n-1}^{(s)}}\right)\right) \cdots\right)\right)\right|_{e^{\Lambda_{0}=e^{\delta}=1}} .
$$

Let $\lambda^{\prime}=\tau_{n-1, n} \lambda$, and set $\boldsymbol{\pi}_{n}^{\prime}=\boldsymbol{\pi}_{n}^{\varepsilon}\left(\lambda^{\prime}, a\right)$. It is easily seen from Theorem 3.1 that

$$
\operatorname{ch} L_{q}\left(\boldsymbol{\pi}_{n-1}\right)=\tau_{n-1, n} \operatorname{ch} L_{q}\left(\boldsymbol{\pi}_{n}^{\prime}\right)
$$

Hence we also obtain the character in the case $s=n-1$.

\section{Remark 3.6.}

(i) It is possible to use other elements of $\widetilde{W}$ in the expression of $\operatorname{ch} L_{q}\left(\boldsymbol{\pi}_{s}\right)$. That is, if $w_{j} \in \widetilde{W}$ $(1 \leq j \leq n-1)$ satisfy $w_{[1, j]} \Lambda_{j}^{(s)}=\xi_{j}^{(s)}$ and $\ell\left(w_{\circ} w_{[1, n-1]}\right)=\ell\left(w_{\circ}\right)+\sum_{j=1}^{n-1} \ell\left(w_{j}\right)$ (here we set $\left.w_{[1, j]}=w_{1} w_{2} \cdots w_{j}\right)$, then it follows that

$$
\operatorname{ch} L_{q}\left(\boldsymbol{\pi}_{s}\right)=\left.\mathcal{D}_{w_{\circ}}\left(e^{\Lambda_{n}^{(s)}} \cdot \mathcal{D}_{w_{1}}\left(e^{\Lambda_{1}^{(s)}} \cdots \mathcal{D}_{w_{n-2}}\left(e^{\Lambda_{n-2}^{(s)}} \cdot \mathcal{D}_{w_{n-1}}\left(e^{\Lambda_{n-1}^{(s)}}\right)\right) \cdots\right)\right)\right|_{e^{\Lambda_{0}=e^{\delta}=1}} .
$$

For example $w_{j}=s_{j-1} s_{j-2} \cdots s_{1} \tau_{0,1}$ satisfy the above conditions when $s=1$. Our choice is made so that the results are stated in a uniform way.

(ii) The right-hand side of the isomorphism (3.1) has a crystal analog, and using this we can express the multiplicities of $L_{q}\left(\boldsymbol{\pi}_{s}\right)$ in terms of crystal bases. For the details, see [21, Corollary 4.11].

Our next result is a formula for multiplicities of simple finite-dimensional $U_{q}(\mathfrak{g})$-modules in $L_{q}\left(\boldsymbol{\pi}_{1}\right)$ which can be deduced from our Theorem 3.2 and the results of [4] and [22]. For that, we prepare a lemma.

Lemma 3.7. Assume that $V$ is a cyclic finite-dimensional $\mathfrak{g}[t]$-module generated by a $\mathfrak{h}$-weight vector $v$, and $\mathfrak{n}_{+}[t] \oplus t \mathfrak{h}[t]$ acts trivially on $v$. Let $\mu \in P^{+}$, and $W$ be the $\mathfrak{g}[t]$-submodule of $V \otimes V(\mu, 0)$ generated by $v \otimes v_{\mu}$, where $v_{\mu}$ denotes a highest weight vector. Then for every $\nu \in P^{+}$, we have

$$
[W: V(\nu+\mu)]=[V: V(\nu)]
$$

where [:] denotes the multiplicity as a $\mathfrak{g}$-module.

Proof. Note that

$$
[W: V(\nu+\mu)]=\operatorname{dim}\left\{w \in W_{\nu+\mu} \mid \mathfrak{n}_{+} w=0\right\}
$$

Since

$$
W=U\left(\mathfrak{n}_{-}[t]\right)\left(v \otimes v_{\mu}\right)=U\left(\mathfrak{n}_{-}\right)\left(U\left(t \mathfrak{n}_{-}[t]\right) v \otimes v_{\mu}\right)
$$

and $W$ is a finite-dimensional $\mathfrak{g}$-module, we see that

$$
\begin{aligned}
& \left\{w \in W_{\nu+\mu} \mid \mathfrak{n}_{+} w=0\right\}=\left\{w \in\left(U\left(t \mathfrak{n}_{-}[t]\right) v \otimes v_{\mu}\right)_{\nu+\mu} \mid \mathfrak{n}_{+} w=0\right\} \\
& \quad=\left\{w \in\left(U\left(t \mathfrak{n}_{-}[t]\right) v\right)_{\nu} \mid \mathfrak{n}_{+} w=0\right\} \otimes v_{\mu}=\left\{w \in\left(U\left(\mathfrak{n}_{-}[t]\right) v\right)_{\nu} \mid \mathfrak{n}_{+} w=0\right\} \otimes v_{\mu} .
\end{aligned}
$$

Hence the assertion follows from (3.2). 
Let $\mathfrak{s p}_{2 n-2}$ be the simple Lie algebra of type $C_{n-1}$, and denote by $P_{\mathfrak{s p}}$ its weight lattice and by $\varpi_{i}^{\mathfrak{s p}}(1 \leq i \leq n-1)$ its fundamental weights. We assume that $\varpi_{i}^{\mathfrak{s p}}$ are labeled as [15, Section 4.8]. Define a map $\iota: P^{+} \rightarrow P_{\mathfrak{s p}}^{+}$by

$$
\iota\left(\sum_{1 \leq i \leq n} \mu_{i} \varpi_{i}\right)=\sum_{1 \leq i \leq n-2} \mu_{i} \varpi_{i}^{\mathfrak{s p}}+\min \left\{\mu_{n-1}, \mu_{n}\right\} \varpi_{n-1}^{\mathfrak{s p}} .
$$

Corollary 3.8. For every $\mu \in P^{+}$, we have

$$
\left[L_{q}\left(\boldsymbol{\pi}_{1}\right): V_{q}(\mu)\right]= \begin{cases}{\left[\mathbf{S}_{\iota(\lambda)}\left(V^{\mathfrak{s p}}\left(\varpi_{1}^{\mathfrak{s p}}\right)\right): V^{\mathfrak{s p}}(\iota(\mu))\right],} & \text { if } \mu_{n}-\mu_{n-1}=\lambda_{n}-\lambda_{n-1}, \\ 0, & \text { otherwise. }\end{cases}
$$

Here $\mathbf{S}_{\nu}\left(\nu \in P_{\mathfrak{s p}}^{+}\right)$denotes the Schur functor (see [22, Section 1]) with respect to the partition $\left(\sum_{j=1}^{n-1} \nu_{j}, \sum_{j=2}^{n-1} \nu_{j}, \ldots, \nu_{n-1}\right)$, and $V^{\mathfrak{s p}}(\nu)$ denotes the simple $\mathfrak{s p}_{2 n-2}$-module with highest weight $\nu$.

Proof. It suffices to show that the right-hand side is equal to $\left[L\left(\boldsymbol{\pi}_{1}\right): V(\mu)\right]$ by Lemma 2.10(iv). Note that Theorem 3.2 and [4, Theorem 1] imply that the graded limit $L\left(\boldsymbol{\pi}_{1}\right)$ is isomorphic to the $\mathfrak{g}[t]$-module " $P(\lambda, 0)^{\Gamma(\lambda, \Psi) "}$ in the notation of [4], where we set $\Psi=\left\{\alpha \in \Delta_{+} \mid\left(\alpha, \varpi_{n-1}+\varpi_{n}\right)=2\right\}$. Then in the case $\lambda_{n-1}=\lambda_{n}$, our assertion is a consequence of [4, Theorem 2] and [22, Theorem 1].

Let us assume $\lambda_{n-1} \neq \lambda_{n}$, and set $\lambda^{\prime}=\lambda_{m}-\lambda_{m^{\prime}}$. We have

$$
L\left(\boldsymbol{\pi}_{1}\right) \cong D\left(w_{\circ} \xi_{1}^{(1)}, \ldots, w_{\circ} \xi_{n}^{(1)}\right)
$$

by Theorem 3.1, and we easily see that $D\left(w_{\circ} \xi_{n}^{(1)}\right) \cong V\left(\lambda^{\prime} \varpi_{m}, 0\right)$ holds. Hence by applying Lemma 3.7 with $V=D\left(w_{\circ} \xi_{1}^{(1)}, \ldots, w_{\circ} \xi_{n-1}^{(1)}\right)$ and $\mu=\lambda^{\prime} \varpi_{m}$, we have for every $\nu \in P^{+}$that

$$
\left[D\left(w_{\circ} \xi_{1}^{(1)}, \ldots, w_{\circ} \xi_{n}^{(1)}\right): V\left(\nu+\lambda^{\prime} \varpi_{m}\right)\right]=\left[D\left(w_{\circ} \xi_{1}^{(1)}, \ldots, w_{\circ} \xi_{n-1}^{(1)}\right): V(\nu)\right],
$$

and the right-hand side is equal to $\left[L\left(\boldsymbol{\pi}_{1}^{\varepsilon}\left(\lambda-\lambda^{\prime} \varpi_{m}, a\right)\right): V(\nu)\right]$ by Theorem 3.1. Hence the assertion is deduced from the case $\lambda_{n-1}=\lambda_{n}$. The proof is complete.

\section{Proofs of main theorems}

Note that the theorems for $s=n-1$ and $s=n$ are equivalent because of the existence of diagram automorphism of $\mathfrak{g}$ interchanging $n-1$ and $n$. Therefore, throughout this section we assume that $s \neq n-1$, and prove the theorems only for the case $s=1, n$. Similarly as the previous section we fix $\lambda=\sum_{i \in I} \lambda_{i} \varpi_{i} \in P^{+}, \varepsilon \in\{ \pm\}$ and $a \in \mathbb{C}^{\times} q^{\mathbb{Z}}$, and write $\boldsymbol{\pi}_{s}=\boldsymbol{\pi}_{s}^{\varepsilon}(\lambda, a)$.

Let $M_{s}(\lambda)$ denote the module defined in Theorem 3.2. We shall prove the existence of three surjective $\mathfrak{g}[t]$-module homomorphisms. More presicely, we prove $M_{s}(\lambda) \rightarrow L\left(\boldsymbol{\pi}_{s}\right)$ in Section 4.1, $D\left(w_{\circ} \xi_{1}^{(s)}, \ldots w_{\circ} \xi_{n}^{(s)}\right) \rightarrow M_{s}(\lambda)$ in Section 4.2, and $L\left(\boldsymbol{\pi}_{s}\right) \rightarrow D\left(w_{\circ} \xi_{1}^{(s)}, \ldots w_{\circ} \xi_{n}^{(s)}\right)$ in Section 4.3. Then both Theorems 3.1 and 3.2 immediately follow from them.

\subsection{Proof for $M_{s}(\lambda) \rightarrow L\left(\pi_{s}\right)$}

Though the proof is similar to that in [18], we will give it for completeness.

Let $v_{\boldsymbol{\pi}_{s}}$ be a highest $\ell$-weight vector of $L_{q}\left(\boldsymbol{\pi}_{s}\right)$, and set $\bar{v}_{\boldsymbol{\pi}_{s}}=1 \otimes v_{\boldsymbol{\pi}_{s}} \in L\left(\boldsymbol{\pi}_{s}\right)$. In order to prove $M_{s}(\lambda) \rightarrow L\left(\boldsymbol{\pi}_{s}\right)$, it is enough to show the relations

$$
\left(f_{\alpha} \otimes t\right) \bar{v}_{\boldsymbol{\pi}_{s}}=0 \quad \text { for } \quad \alpha \in \Delta_{+}^{(s)},
$$

since the other relations hold by Lemma 2.10(ii). 
Let $r \in S \backslash\{s\}$ and $J=I \backslash I_{r}$. Then the subalgebra $U_{q}\left(\mathbf{L g}_{J}\right) \subseteq U_{q}(\mathbf{L g})$ is a quantum loop algebra of type $A$. By [10, Lemma 2.3], the $U_{q}\left(\mathbf{L g}_{J}\right)$-submodule of $L_{q}\left(\boldsymbol{\pi}_{s}\right)$ generated by $v_{\boldsymbol{\pi}_{s}}$ is isomorphic to the simple $U_{q}\left(\mathbf{L g}_{J}\right)$-module with highest $\ell$-weight $\left(\left(\boldsymbol{\pi}_{s}\right)_{i}(u)\right)_{i \in J}$. Denote this $U_{q}\left(\mathbf{L g}_{J}\right)$-submodule by $L_{q}^{\prime}$. Then we see from Remark 2.7 and [10, Theorem 3.1] that $L_{q}^{\prime}$ is also simple as a $U_{q}\left(\mathfrak{g}_{J}\right)$-module. From this and the construction of graded limits, it follows that the $\mathbf{L} \mathfrak{g}_{J}$-submodule

$$
L^{\prime}=U\left(\mathbf{L} \mathfrak{g}_{J}\right) \bar{v}_{\boldsymbol{\pi}_{s}} \subseteq L\left(\boldsymbol{\pi}_{s}\right)
$$

is simple as a $\mathfrak{g}_{J}$-module. Hence the restriction of the surjective homomorphism $L\left(\boldsymbol{\pi}_{s}\right) \rightarrow V(\lambda, 0)$ in Lemma 2.10(i) to $L^{\prime}$ is an isomorphism, which obviously implies $\left(\mathfrak{g}_{J} \otimes t\right) \bar{v}_{\pi_{s}}=0$. Now the relations (4.1) obviously follow from the definition of $\Delta_{+}^{(s)}$. The proof is complete.

\section{$4.2 \quad$ Proof for $D\left(w_{\circ} \xi_{1}^{(s)}, \ldots, w_{\circ} \xi_{n}^{(s)}\right) \rightarrow M_{s}(\lambda)$}

Throughout this subsection, we assume that $s \in\{1, n\}$ is fixed. Note that some notation appearing below may depend on $s$ though it is not written explicitly.

Let us prepare several notation. For $1 \leq p \leq q \leq n$, set

$$
\alpha_{p, q}= \begin{cases}\alpha_{p}+\alpha_{p+1}+\cdots+\alpha_{q}, & q \leq n-1, \\ \alpha_{p}+\alpha_{p+1}+\cdots+\alpha_{n-2}+\alpha_{n}, & q=n .\end{cases}
$$

Note that

$$
\Delta_{+}=\left\{\alpha_{p, q} \mid p \leq q,(p, q) \neq(n-1, n)\right\} \sqcup\left\{\alpha_{p, n}+\alpha_{q, n-1} \mid p<q<n\right\} .
$$

Set $\sigma_{i}=s_{i} s_{i+1} \cdots s_{n-1} \in \widehat{W}$ for $1 \leq i \leq n$ and $\sigma_{0}=\tau_{0,1} \tau_{n-1, n} \sigma_{1}=\sigma$. For $0 \leq i \leq n$ and $1 \leq j \leq n-1$, define $\rho_{i, j}: \widehat{\Delta} \rightarrow \mathbb{Z}_{\geq 0}$ by

$$
\rho_{i, j}(\alpha)=\sum_{k=j}^{n-1} \max \left\{0,-\left(\alpha, \sigma_{i} \sigma^{k-j} \Lambda_{k}^{(s)}\right)\right\} .
$$

When $j<n-1$, we have

$$
\rho_{n, j}(\alpha)=\rho_{0, j+1}(\alpha)+\max \left\{0,-\left(\alpha, \Lambda_{j}^{(s)}\right)\right\}=\rho_{0, j+1}(\alpha) \quad \text { for } \quad \alpha \in \widehat{\Delta}_{+}^{\mathrm{re}}
$$

since $\Lambda_{j}^{(s)} \in \widehat{P}^{+}$.

Lemma 4.1. Let $1 \leq i \leq n$ and $1 \leq j \leq n-1$, and assume that $\alpha=\beta+k \delta \in \widehat{\Delta}_{+}^{\text {re }}$ satisfies $\rho_{i, j}(\alpha)>0$.

(i) If $i=n$, we have

$$
\beta \in\left\{-\alpha_{p, n-1} \mid p<n\right\} \sqcup\left\{-\left(\alpha_{p, n}+\alpha_{q, n-1}\right) \mid p<q<n\right\} .
$$

(ii) If $1 \leq i \leq n-1$, we have

$$
\begin{aligned}
\beta \in\left\{\alpha_{i, q} \mid i \leq q<n\right\} \sqcup\left\{-\alpha_{p, i-1} \mid p<i\right\} \sqcup\left\{-\alpha_{p, n} \mid p \neq i\right\} \\
\sqcup\left\{-\left(\alpha_{p, n}+\alpha_{q, n-1}\right) \mid p<q<n, p \neq i, q \neq i\right\} .
\end{aligned}
$$


Proof. In both the cases $s=1$ and $s=n$, it follows from Lemma 3.4(i) that

$$
\sigma^{k-j} \Lambda_{k}^{(s)} \in \sum_{0 \leq p \leq n} \mathbb{Z}_{\geq 0}\left(\varpi_{p}+\Lambda_{0}\right)+\sum_{1 \leq p \leq n} \mathbb{Z}_{\geq 0}\left(\varpi_{p}+\varpi_{n-1}+\Lambda_{0}\right)
$$

holds for every $1 \leq j \leq k \leq n-1$. Hence $\rho_{n, j}(\alpha)>0$ implies $\beta \in-\Delta_{+}$and $k \geq 1$, and if $\beta=-\sum_{i \in I} t_{i} \alpha_{i}$ then we have

$$
t_{p}+t_{n-1}>k \geq 1 \quad \text { for some } \quad 1 \leq p \leq n .
$$

This immediately implies the assertion (i). Note that $\rho_{i, j}(\alpha)=\rho_{i+1, j}\left(s_{i} \alpha\right)$ holds for $1 \leq i \leq n-1$ by the definition of $\rho_{i, j}$. Hence $\rho_{i, j}(\alpha)>0$ implies that we have either $\alpha=\alpha_{i}$ or $\alpha=s_{i} \gamma$ for some $\gamma \in \widehat{\Delta}_{+}^{\text {re }}$ such that $\rho_{i+1, j}(\gamma)>0$. From this, the assertion (ii) is easily proved by the descending induction on $i$.

For $0 \leq i \leq n$ and $1 \leq j \leq n-1$, set

$$
\begin{aligned}
& D(i, j)=D\left(\sigma_{i} \Lambda_{j}^{(s)}, \sigma_{i} \sigma \Lambda_{j+1}^{(s)}, \ldots, \sigma_{i} \sigma^{n-j-1} \Lambda_{n-1}^{(s)}\right), \quad \text { and } \\
& v(i, j)=v_{\sigma_{i} \Lambda_{j}^{(s)}} \otimes v_{\sigma_{i} \sigma \Lambda_{j+1}^{(s)}} \otimes \cdots \otimes v_{\sigma_{i} \sigma^{n-j-1} \Lambda_{n-1}^{(s)}} \in D(i, j) .
\end{aligned}
$$

For $\alpha=\beta+k \delta \in \widehat{\Delta}^{\text {re }}$ with $\beta \in \Delta$ and $k \in \mathbb{Z}$, denote by $x_{\alpha} \in \widehat{\mathfrak{g}}$ the vector $e_{\beta} \otimes t^{k}$. For $i \in \widehat{I}$, define a Lie subalgebra $\widehat{\mathfrak{n}}_{i}$ of $\widehat{\mathfrak{n}}_{+}$by

$$
\widehat{\mathfrak{n}}_{i}=\bigoplus_{\alpha \in \widehat{\Delta}_{+}^{\mathrm{re}} \backslash\left\{\alpha_{i}\right\}} \mathbb{C} x_{\alpha} \oplus t \mathfrak{h}[t]
$$

We shall determine the generators of the annihilators $\operatorname{Ann}_{U\left(\widehat{\mathfrak{n}}_{+}\right)} v(i, j)$ inductively, along the lines of [21, Section 5.1]. For that, we need the following lemma which is proved in [14, Section 3] (see also [21, Lemma 5.3]).

Lemma 4.2. Let $V$ be an integrable $\widehat{\mathfrak{g}}$-module, $T$ a finite-dimensional $\widehat{\mathfrak{b}}$-submodule of $V, i \in \widehat{I}$ and $\xi \in \widehat{P}$ such that $\left(\alpha_{i}, \xi\right) \geq 0$. Assume that the following conditions hold:

(i) $T$ is generated by a $\widehat{\mathfrak{h}}$-weight vector $v \in T_{\xi}$ satisfying $e_{i} v=0$.

(ii) There is an $\operatorname{ad}\left(e_{i}\right)$-invariant left $U\left(\widehat{\mathfrak{n}}_{i}\right)$-ideal $\mathcal{I}$ such that

$$
\operatorname{Ann}_{U\left(\widehat{\mathfrak{n}}_{+}\right)} v=U\left(\widehat{\mathfrak{n}}_{+}\right) e_{i}+U\left(\widehat{\mathfrak{n}}_{+}\right) \mathcal{I}
$$

(iii) We have $\operatorname{ch}_{\widehat{\mathfrak{h}}} F_{i} T=\mathcal{D}_{i} \operatorname{ch}_{\widehat{\mathfrak{h}}} T$.

Let $v^{\prime}=f_{i}^{\left(\alpha_{i}, \xi\right)} v$. Then we have

$$
\operatorname{Ann}_{U\left(\widehat{\mathfrak{n}}_{+}\right)} v^{\prime}=U\left(\widehat{\mathfrak{n}}_{+}\right) e_{i}^{\left(\alpha_{i}, \xi\right)+1}+U\left(\widehat{\mathfrak{n}}_{+}\right) r_{i}(\mathcal{I}),
$$

where $r_{i}$ denotes the algebra automorphism of $U(\widehat{\mathfrak{g}})$ corresponding to the reflection $s_{i}$.

Proposition 4.3. The following assertion $\left(\mathrm{A}_{i, j}\right)$ holds for every $0 \leq i \leq n$ and $1 \leq j \leq n-1$

$$
\left(\mathrm{A}_{i, j}\right) \quad \operatorname{Ann}_{U\left(\widehat{\mathfrak{n}}_{+}\right)} v(i, j)=U\left(\widehat{\mathfrak{n}}_{+}\right)\left(\sum_{\alpha \in \widehat{\Delta}_{+}^{\mathrm{re}}} \mathbb{C} x_{\alpha}^{\rho_{i, j}(\alpha)+1}+t \mathfrak{h}[t]\right) .
$$


Proof. We will prove the assertion by the descending induction on $(i, j)$. The assertion $\left(\mathrm{A}_{n, n-1}\right)$ is obvious since $D(n, n-1)=\mathbb{C} v(n, n-1)$ is a trivial $\widehat{\mathfrak{n}}_{+}$-module and $\rho_{n, n-1}(\alpha)=0$ for all $\alpha \in \widehat{\Delta}_{+}^{\mathrm{re}}$. Since

$$
v(n, j)=v_{\Lambda_{j}^{(s)}} \otimes v(0, j+1),
$$

we easily see that $\left(\mathrm{A}_{0, j+1}\right)$ implies $\left(\mathrm{A}_{n, j}\right)$ by $(4.2)$, and $\left(\mathrm{A}_{1, j}\right)$ implies $\left(\mathrm{A}_{0, j}\right)$ since

$$
D(0, j) \cong F_{\tau_{0,1} \tau_{n-1, n}} D(1, j) \quad \text { and } \quad \rho_{0, j}(\alpha)=\rho_{1, j}\left(\tau_{0,1} \tau_{n-1, n} \alpha\right) .
$$

It remains to show that $\left(\mathrm{A}_{i, j}\right)$ implies $\left(\mathrm{A}_{i-1, j}\right)$ when $2 \leq i \leq n$. Let $\xi(i, j)=\sum_{k=j}^{n-1} \sigma_{i} \sigma^{k-j} \Lambda_{k}^{(s)} \in \widehat{P}$, which is the weight of $v(i, j)$. Since $\left(\alpha_{i-1}, \sigma_{i} \sigma^{k-j} \Lambda_{k}^{(s)}\right) \geq 0$ holds for all $k \geq j$ by Lemma 3.4(ii), we have

$$
e_{i-1} v(i, j)=0 \quad \text { and } \quad f_{i-1}^{\left(\alpha_{i-1}, \xi(i, j)\right)} v(i, j) \in \mathbb{C}^{\times} v(i-1, j) .
$$

In addition, we have $\rho_{i-1, j}(\alpha)=\rho_{i, j}\left(s_{i-1} \alpha\right)$ for $\alpha \in \widehat{\Delta}_{+}^{\text {re }}$ and in particular

$$
\rho_{i-1, j}\left(\alpha_{i-1}\right)=\rho_{i, j}\left(-\alpha_{i-1}\right)=\left(\alpha_{i-1}, \xi(i, j)\right) .
$$

Therefore, it suffices to show the ad $\left(e_{i-1}\right)$-invariance of the left $U\left(\widehat{\mathfrak{n}}_{i-1}\right)$-ideal

$$
\mathcal{I}_{i, j}=U\left(\widehat{\mathfrak{n}}_{i-1}\right)\left(\sum_{\alpha \in \widehat{\Delta}_{+}^{\mathrm{re}} \backslash\left\{\alpha_{i-1}\right\}} \mathbb{C} x_{\alpha, j}^{\rho_{i, j}(\alpha)+1}+t \mathfrak{h}[t]\right)
$$

by Lemma 4.2 (note that the condition (iii) holds by Proposition 2.2). Since $\rho_{i, j}\left(\alpha_{i-1}+\mathbb{Z}_{>0} \delta\right)=0$ holds by Lemma 4.1, we have

$$
\left[e_{i-1}, t \mathfrak{h}[t]\right]=e_{i-1} \otimes t \mathbb{C}[t] \subseteq \mathcal{I}_{i, j}
$$

Hence it is enough to verify that

$$
\left[e_{i-1}, x_{\alpha}^{\rho_{i, j}(\alpha)+1}\right] \in \mathcal{I}_{i, j}
$$

for every $\alpha \in \widehat{\Delta}_{+}^{\text {re }} \backslash\left\{\alpha_{i-1}\right\}$. If $\alpha=-\alpha_{i-1}+k \delta(k>0)$, then (4.3) follows from $t \mathfrak{h}[t] \oplus e_{i-1} \otimes t \mathbb{C}[t] \subseteq$ $\mathcal{I}_{i, j}$. Hence we may assume that $\alpha$ satisfies $\left[\left[e_{i-1}, x_{\alpha}\right], x_{\alpha}\right]=0$. If $\left[e_{i-1}, x_{\alpha}\right]=0,(4.3)$ is obvious, and otherwise we have

$$
\left[e_{i-1}, x_{\alpha}^{\rho_{i, j}(\alpha)+1}\right] \in \mathbb{C} x_{\alpha}^{\rho_{i, j}(\alpha)} x_{\alpha+\alpha_{i-1}} .
$$

It is directly checked from Lemma 4.1 that if $\beta \in \widehat{\Delta}_{+}^{\text {re }}$ satisfies $\beta-\alpha_{i-1} \in \widehat{\Delta}_{+}^{\text {re }}$, then $\rho_{i, j}(\beta)=0$. Hence we have $\rho_{i, j}\left(\alpha+\alpha_{i-1}\right)=0$, and (4.3) follows. The proof is complete.

In the sequel we write $\rho=\rho_{0,1}$ for brevity. Note that we have

$$
\rho(\alpha)=\sum_{1 \leq j \leq n-1} \max \left\{0,-\left(\alpha, \xi_{j}^{(s)}\right)\right\}
$$

The following assertions are proved from the definition of $\xi_{j}^{(s)}$,s by a direct calculation.

(i) Assume that $s=1$. 
(a) For $\beta+k \delta \in \widehat{\Delta}_{+}^{\mathrm{re}}, \rho(\beta+k \delta)=0$ holds unless

$$
-\beta \in\left\{\alpha_{p, n}+\alpha_{q, n-1} \mid p<q<n\right\} \quad \text { and } \quad k=1 .
$$

(b) We have

$$
\rho\left(-\left(\alpha_{p, n}+\alpha_{q, n-1}\right)+\delta\right)=\sum_{j=q}^{n-2} \lambda_{j}+\lambda_{m^{\prime}} .
$$

(ii) Assume that $s=n$.

(a) For $\beta+k \delta \in \widehat{\Delta}_{+}^{\mathrm{re}}, \rho(\beta+k \delta)=0$ holds unless

$$
\begin{aligned}
& -\beta \in\left\{\alpha_{p, n-1} \mid p \leq n-3\right\} \quad \text { and } \quad k=1, \quad \text { or } \\
& -\beta \in\left\{\alpha_{p, n}+\alpha_{q, n-1} \mid p<q<n\right\} \quad \text { and } \quad k=1,2 .
\end{aligned}
$$

(b) We have

$$
\begin{aligned}
& \rho\left(-\alpha_{p, n-1}+\delta\right)=\min \left\{\sum_{j=p}^{n-3} \lambda_{j}, \lambda_{n-1}\right\}, \quad \text { and } \\
& \rho\left(-\left(\alpha_{p, n}+\alpha_{q, n-1}\right)+k \delta\right)= \begin{cases}\min \left\{\sum_{j=p}^{n-3} \lambda_{j}, \lambda_{n-1}\right\}+\sum_{j=q}^{n-2} \lambda_{j}, & \text { if } k=1, \\
\min \left\{\sum_{j=q}^{n-3} \lambda_{j}, \lambda_{n-1}\right\}, & \text { if } \quad k=2 .\end{cases}
\end{aligned}
$$

Set $D=D\left(\xi_{n}^{(s)}, \xi_{1}^{(s)}, \ldots, \xi_{n-1}^{(s)}\right)$ and $v_{D}=v_{\xi_{n}^{(s)}} \otimes v(0,1) \in D$. By Proposition 4.3, we have

$$
\operatorname{Ann}_{U\left(\widehat{\mathfrak{n}}_{+}\right)} v_{D}=\operatorname{Ann}_{U\left(\widehat{\mathfrak{n}}_{+}\right)} v(0,1)=U\left(\widehat{\mathfrak{n}}_{+}\right)\left(\sum_{\alpha \in \widehat{\Delta}_{+}^{\mathrm{re}}} \mathbb{C} x_{\alpha}^{\rho(\alpha)+1}+t \mathfrak{h}[t]\right) .
$$

Let $v_{M} \in M_{s}(\lambda)$ denote the generator in the definition. The proof of the following lemma is elementary (see, e.g., [20, Lemma 4.5]).

Lemma 4.4. If $\beta, \gamma \in \widehat{\Delta}_{+}^{\text {re }}$ satisfy $(\beta, \gamma)=-1, x_{\beta}^{b+1} v_{M}=0$ and $x_{\gamma}^{c+1} v_{M}=0$ with $b, c \in \mathbb{Z}_{\geq 0}$, then $x_{\beta+\gamma}^{b+c+1} v_{M}=0$ holds.

Lemma 4.5. There exists a $\left(\mathfrak{h} \oplus \widehat{\mathfrak{n}}_{+}\right)$-module homomorphism from $D$ to $M_{s}(\lambda)$ mapping $v_{D}$ to $v_{M}$.

Proof. Since $t \mathfrak{h}[t] v_{M}=0$ and the $\mathfrak{h}$-weights of both $v_{D}$ and $v_{M}$ are $\lambda$, it suffices to show $x_{\alpha}^{\rho(\alpha)+1} v_{M}=0$ for all $\alpha \in \widehat{\Delta}_{+}^{\text {re }}$.

First we consider the case $s=1$. The assertion for $\alpha=-\left(\alpha_{p, n}+\alpha_{q, n-1}\right)+\delta$ with $p<q<n$ is proved by applying Lemma 4.4 with $\beta=-\alpha_{p, m}+\delta$ and $\gamma=-\alpha_{q, m^{\prime}}$. The assertion for remaining $\alpha \in \widehat{\Delta}_{+}^{\text {re }}$ is easily proved from the defining relations of $M_{1}(\lambda)$.

Next we consider the case $s=n$. If $\sum_{j=p}^{n-3} \lambda_{j} \geq \lambda_{n-1}$, the assertion for $\alpha=-\alpha_{p, n-1}+\delta$ is proved by applying the lemma with $\beta=-\alpha_{p, n-2}+\delta, \gamma=-\alpha_{n-1}$. Otherwise it is proved by applying the lemma with $\beta=-\alpha_{p, n-3}, \gamma=-\alpha_{n-2, n-1}+\delta$. The assertion for $\alpha=-\left(\alpha_{p, n}+\alpha_{n-1}\right)+\delta$ is similarly proved, and then the assertion for $\alpha=-\left(\alpha_{p, n}+\alpha_{q, n-1}\right)+\delta$ is verified by applying the lemma with $\beta=-\left(\alpha_{p, n}+\alpha_{n-1}\right)+\delta$ and $\gamma=-\alpha_{q, n-2}$. Finally the assertion for $\alpha=-\left(\alpha_{p, n}+\alpha_{q, n-1}\right)+2 \delta$ is shown by applying the lemma with $\beta=-\alpha_{p, n}+\delta$ and $\gamma=-\alpha_{q, n-1}+\delta$. The assertion for remaining $\alpha \in \widehat{\Delta}_{+}^{\text {re }}$ is easily proved from the defining relations of $M_{n}(\lambda)$. 
Now we can prove the existence of a surjective $\mathfrak{g}[t]$-module homomorphism

$$
D\left(w_{\circ} \xi_{n}^{(s)}, w_{\circ} \xi_{1}^{(s)}, \ldots, w_{\circ} \xi_{n-1}^{(s)}\right) \rightarrow M_{s}(\lambda)
$$

by exactly the same arguments with [21, two paragraphs below Lemma 5.2] from Lemma 4.5. Since $D\left(w_{\circ} \xi_{n}^{(s)}, w_{\circ} \xi_{1}^{(s)}, \ldots w_{\circ} \xi_{n-1}^{(s)}\right) \cong D\left(w_{\circ} \xi_{1}^{(s)}, \ldots, w_{\circ} \xi_{n}^{(s)}\right)$ holds by definition, the proof is complete.

\subsection{Proof for $L\left(\pi_{s}\right) \rightarrow D\left(w_{\circ} \xi_{1}^{(s)}, \ldots, w_{\circ} \xi_{n}^{(s)}\right)$}

We shall prove the assertion in the case $\varepsilon=+$ (the case $\varepsilon=-$ is similarly proved). For $i \in I$, set

$$
p_{i}= \begin{cases}n-2-i, & i \leq n-2, \\ 1, & i=n-1, n,\end{cases}
$$

which is the distance between the nodes $i$ and $n-2$ in the Dynkin diagram. We need the following lemma that is proved from [3].

\section{Lemma 4.6 .}

(i) Let $i_{1}, \ldots, i_{p} \in I, b_{1}, \ldots, b_{p} \in \mathbb{C}(q)^{\times}$and $l_{1}, \ldots, l_{p} \in \mathbb{Z}_{>0}$, and assume that

$$
b_{r} \notin q^{\mathbb{Z}>0} b_{s} q^{l_{r}-l_{s}+\left|p_{i_{r}}-p_{i_{s}}\right|} \quad \text { for all } \quad r<s .
$$

Then the submodule of $L_{q}\left(\boldsymbol{\pi}_{l_{1}, b_{1}}^{\left(i_{1}\right)}\right) \otimes \cdots \otimes L_{q}\left(\boldsymbol{\pi}_{l_{p}, b_{p}}^{\left(i_{p}\right)}\right)$ generated by the tensor product of highest $\ell$-weight vectors is isomorphic to $L_{q}\left(\prod_{k=1}^{p} \pi_{l_{k}, b_{k}}^{\left(i_{k}\right)}\right)$.

(ii) If $i, j \in I, b \in \mathbb{C}(q)^{\times}, l \in \mathbb{Z}_{>0}$ and $-\left|p_{i}-p_{j}\right| \leq k \leq\left|p_{i}-p_{j}\right|$, then $L_{q}\left(\boldsymbol{\pi}_{l, b}^{(i)}\right) \otimes L_{q}\left(\boldsymbol{\pi}_{l, b q^{k}}^{(j)}\right)$ is simple.

Proof. (i) For $r<s$, it is directly checked that $L_{q}\left(\boldsymbol{\pi}_{l_{s}, b_{s}}^{\left(i_{s}\right)}\right) \otimes L_{q}\left(\boldsymbol{\pi}_{l_{r}, b_{r}}^{\left(i_{r}\right)}\right)$ satisfies the condition of [3, Corollary 6.2], which assures that the module is generated by the tensor product of highest $\ell$-weight vectors. Hence

$$
L_{q}\left(\boldsymbol{\pi}_{l_{p}, b_{p}}^{\left(i_{p}\right)}\right) \otimes L_{q}\left(\boldsymbol{\pi}_{l_{p-1}, b_{p-1}}^{\left(i_{p-1}\right)}\right) \otimes \cdots \otimes L_{q}\left(\boldsymbol{\pi}_{l_{1}, b_{1}}^{\left(i_{1}\right)}\right)
$$

is also generated by the tensor product of highest $\ell$-weight vectors (see [3, sentences above Corollary 6.2]). Now the assertion (i) follows by dualizing the statement and applying Lemma 2.4 (the bijection $i \mapsto \bar{i}$ in the lemma is $\tau_{n-1, n}$ ). (ii) We see from the above argument that $L_{q}\left(\boldsymbol{\pi}_{l, b}^{(i)}\right) \otimes$ $L_{q}\left(\boldsymbol{\pi}_{l, b q^{k}}^{(j)}\right)$ is both cyclic and cocyclic, and hence simple.

Lemma 4.7. Let $i \in I \backslash\{n-2, n-1\}, b \in \mathbb{C}^{\times} q^{\mathbb{Z}}$ and $l \in \mathbb{Z}_{\geq 0}$. The graded limit $L\left(\boldsymbol{\pi}_{l, b}^{(i)} \boldsymbol{\pi}_{l, b q^{p-1}}^{(n-1)}\right)$ is isomorphic to $D\left(l w_{\circ}\left(\varpi_{i}+\varpi_{n-1}+\Lambda_{0}\right)\right)$ as a $\mathfrak{g}[t]$-module.

Proof. Note that

$$
\boldsymbol{\pi}_{l, b}^{(i)} \boldsymbol{\pi}_{l, b q^{p_{i}-1}}^{(n-1)}= \begin{cases}\boldsymbol{\pi}_{n}^{+}\left(l\left(\varpi_{i}+\varpi_{n-1}\right), b q^{\left(\delta_{i 1}-1\right) l-i+1}\right) & \text { if } \quad i \leq n-3, \\ \boldsymbol{\pi}_{1}^{+}\left(l\left(\varpi_{n}+\varpi_{n-1}\right), b q^{-l-n+2}\right) & \text { if } \quad i=n .\end{cases}
$$

Hence by Sections 4.1 and 4.2, there exists a surjective homomorphism

$$
D\left(l w_{\circ}\left(\varpi_{i}+\varpi_{n-1}+\Lambda_{0}\right)\right) \rightarrow L\left(\boldsymbol{\pi}_{l, b}^{(i)} \boldsymbol{\pi}_{l, b q^{p_{i}-1}}^{(n-1)}\right) .
$$


Therefore it suffices to show the equality of the dimensions. By Lemma 4.6(ii), we have

$$
L_{q}\left(\boldsymbol{\pi}_{l, b}^{(i)} \boldsymbol{\pi}_{l, b q^{p_{i}-1}}^{(n-1)}\right) \cong L_{q}\left(\boldsymbol{\pi}_{l, b}^{(i)}\right) \otimes L_{q}\left(\boldsymbol{\pi}_{l, b q^{p_{i}-1}}^{(n-1)}\right),
$$

which implies

$$
\begin{array}{rl}
\operatorname{dim}_{\mathbb{C}} & L\left(\boldsymbol{\pi}_{l, b}^{(i)} \boldsymbol{\pi}_{l, b q^{p_{i}-1}}^{(n-1)}\right)=\operatorname{dim}_{\mathbb{C}(q)} L_{q}\left(\boldsymbol{\pi}_{l, b}^{(i)} \boldsymbol{\pi}_{l, b q^{p_{i}-1}}^{(n-1)}\right) \\
& =\operatorname{dim}_{\mathbb{C}(q)} L_{q}\left(\boldsymbol{\pi}_{l, b}^{(i)}\right) \cdot \operatorname{dim}_{\mathbb{C}(q)} L_{q}\left(\boldsymbol{\pi}_{l, b q^{p_{i}-1}}^{(n-1)}\right)=\operatorname{dim}_{\mathbb{C}} L\left(\boldsymbol{\pi}_{l, b}^{(i)}\right) \cdot \operatorname{dim}_{\mathbb{C}} L\left(\boldsymbol{\pi}_{l, b q^{p_{i}-1}}^{(n-1)}\right) \\
& =\operatorname{dim}_{\mathbb{C}} D\left(l w_{\circ}\left(\varpi_{i}+\Lambda_{0}\right)\right) \cdot \operatorname{dim}_{\mathbb{C}} D\left(l w_{\circ}\left(\varpi_{n-1}+\Lambda_{0}\right)\right),
\end{array}
$$

where the last equality follows from [5, Proposition 5.1.3]. On the other hand, we have

$$
\operatorname{dim}_{\mathbb{C}} D\left(l w_{\circ}\left(\varpi_{i}+\varpi_{n-1}+\Lambda_{0}\right)\right)=\operatorname{dim}_{\mathbb{C}} D\left(l w_{\circ}\left(\varpi_{i}+\Lambda_{0}\right)\right) \cdot \operatorname{dim}_{\mathbb{C}} D\left(l w_{\circ}\left(\varpi_{n-1}+\Lambda_{0}\right)\right)
$$

by [11, Theorem 1]. Hence the assertion is proved.

Now let us begin the proof of the assertion $L\left(\boldsymbol{\pi}_{s}\right) \rightarrow D\left(w_{\circ} \xi_{1}^{(s)}, \ldots, w_{\circ} \xi_{n}^{(s)}\right)$. First we prove this in the case $s=n$. Let $\left(a_{i}\right)_{i \in I}$ be the sequence in the definition of $\boldsymbol{\pi}_{n}=\boldsymbol{\pi}_{n}^{+}(\lambda, a)$, and define $U_{q}(\mathbf{L} \mathfrak{g})$-modules $L_{q}[j]$ for $1 \leq j \leq n-2$ and $j=n$ by

$$
L_{q}[j]=\left\{\begin{array}{lll}
L_{q}\left(\boldsymbol{\pi}_{\lambda_{j}, a_{j}}^{(j)}\right), & \text { if } j<\ell, \quad j=n-2, n, \\
L_{q}\left(\boldsymbol{\pi}_{\lambda_{\ell}-\bar{\lambda}, a_{\ell} q^{-\bar{\lambda}}}^{(\ell)}\right) \otimes L_{q}\left(\boldsymbol{\pi}_{\bar{\lambda}, a_{\ell} q^{\lambda_{\ell}-\bar{\lambda}}}^{(\ell)} \boldsymbol{\pi}_{\bar{\lambda}, a_{\ell} q^{\lambda_{\ell}-\bar{\lambda}+p_{\ell}-1}}^{(n-1)}\right), & \text { if } j=\ell, \\
L_{q}\left(\boldsymbol{\pi}_{\bar{\lambda}, a_{1} q^{-\lambda_{1}-\bar{\lambda}+n-4}}^{(n-1)}\right) \otimes L_{q}\left(\boldsymbol{\pi}_{\lambda_{1}, a_{1}}^{(1)} \boldsymbol{\pi}_{\left.\lambda_{1}, a_{1} q^{n-4}\right),}^{(n-1)}\right) & \text { if } \ell=0, \quad j=1, \\
L_{q}\left(\boldsymbol{\pi}_{\lambda_{j}, a_{j}}^{(j)} \boldsymbol{\pi}_{\lambda_{j}, a_{j} q^{p_{j}-1}}^{(n-1)}\right), & \text { otherwise. }
\end{array}\right.
$$

There exists an injective $U_{q}(\mathbf{L} \mathfrak{g})$-module homomorphism

$$
L_{q}\left(\boldsymbol{\pi}_{n}\right) \hookrightarrow L_{q}[1] \otimes \cdots \otimes L_{q}[n-2] \otimes L_{q}[n]
$$

by Lemma 4.6, and this induces a $U_{\mathbf{A}}(\mathbf{L} \mathfrak{g})$-module homomorphism

$$
L_{\mathbf{A}}\left(\boldsymbol{\pi}_{n}\right) \rightarrow L_{\mathbf{A}}[1] \otimes \cdots \otimes L_{\mathbf{A}}[n-2] \otimes L_{\mathbf{A}}[n],
$$

where we set

$$
L_{\mathbf{A}}[j]=\left\{\begin{array}{lll}
L_{\mathbf{A}}(\boldsymbol{\pi}), & \text { if } & L_{q}[j]=L_{q}(\boldsymbol{\pi}), \\
L_{\mathbf{A}}\left(\boldsymbol{\pi}^{1}\right) \otimes L_{\mathbf{A}}\left(\boldsymbol{\pi}^{2}\right), & \text { if } & L_{q}[j]=L_{q}\left(\boldsymbol{\pi}^{1}\right) \otimes L_{q}\left(\boldsymbol{\pi}^{2}\right) .
\end{array}\right.
$$

Applying $\mathbb{C} \otimes_{\mathbf{A}}-$ and taking the pull-back with respect to the automorphism $\varphi_{\bar{a}}$, we obtain a $\mathfrak{g}[t]$-module homomorphism $L\left(\boldsymbol{\pi}_{n}\right) \rightarrow \bigotimes_{j} L[j]$, where $L[j]$ denotes the graded limit or the tensor product of the two graded limits. Note that, by construction, this homomorphism maps a highest weight vector of $L\left(\boldsymbol{\pi}_{n}\right)$ to the tensor product of highest weight vectors. By Lemma 4.7 and [5, Proposition 5.1.3], we have

$$
L[j] \cong \begin{cases}D\left(\left(\lambda_{\ell}-\bar{\lambda}\right) w_{\circ}\left(\varpi_{\ell}+\Lambda_{0}\right)\right) \otimes D\left(\bar{\lambda} w_{\circ}\left(\varpi_{\ell}+\varpi_{n-1}+\Lambda_{0}\right)\right), & \text { if } j=\ell, \\ D\left(\bar{\lambda} w_{\circ}\left(\varpi_{n-1}+\Lambda_{0}\right)\right) \otimes D\left(\lambda_{1} w_{\circ}\left(\varpi_{1}+\varpi_{n-1}+\Lambda_{0}\right)\right), & \text { if } \ell=0, \quad j=1, \\ D\left(w_{\circ} \xi_{j}^{(n)}\right), & \text { otherwise. }\end{cases}
$$

Hence in order to complete the proof, it suffices to show that

$$
\begin{aligned}
& D\left(\left(\lambda_{\ell}-\bar{\lambda}\right) w_{\circ}\left(\varpi_{\ell}+\Lambda_{0}\right), \bar{\lambda} w_{\circ}\left(\varpi_{\ell}+\varpi_{n-1}+\Lambda_{0}\right)\right) \cong D\left(w_{\circ} \xi_{\ell}^{(n)}\right), \quad \text { and } \\
& D\left(\bar{\lambda} w_{\circ}\left(\varpi_{n-1}+\Lambda_{0}\right), \lambda_{1} w_{\circ}\left(\varpi_{1}+\varpi_{n-1}+\Lambda_{0}\right)\right) \cong D\left(w_{\circ} \xi_{1}^{(n)}\right) \quad \text { when } \quad \ell=0 .
\end{aligned}
$$


The first isomorphism follows since we have

$$
\begin{aligned}
D\left(w_{\circ} \xi_{\ell}^{(n)}\right) & \cong F_{w_{\circ} \sigma^{\ell}}\left(\Lambda_{\ell}^{(n)}\right) \cong F_{w_{\circ} \sigma^{\ell}}\left(D\left(\left(\lambda_{\ell}-\bar{\lambda}\right) \Lambda_{0}\right) \otimes D\left(\bar{\lambda}\left(\varpi_{n-1}+\Lambda_{0}\right)\right)\right) \\
& \cong D\left(\left(\lambda_{\ell}-\bar{\lambda}\right) w_{\circ}\left(\varpi_{\ell}+\Lambda_{0}\right), \bar{\lambda} w_{\circ}\left(\varpi_{\ell}+\varpi_{n-1}+\Lambda_{0}\right)\right)
\end{aligned}
$$

by Proposition 2.1, and the second one is also proved similarly. The proof for $s=n$ is complete.

The case $s=1$ can be proved by a similar (and simpler) argument in which we replace the definition of $L_{q}[i]$ given in (4.4) by the following:

$$
L_{q}[j]= \begin{cases}L_{q}\left(\boldsymbol{\pi}_{\lambda_{j}, a_{j}}^{(j)}\right), & j \leq n-2, \\ L_{q}\left(\boldsymbol{\pi}_{\lambda_{m^{\prime}}, a_{m^{\prime}}}^{\left(m^{\prime}\right)} \boldsymbol{\pi}_{\lambda_{m^{\prime}}, a_{m^{\prime}}}^{(m)}\right), & j=n-1, \\ L_{q}\left(\boldsymbol{\pi}_{\lambda_{m}-\lambda_{m^{\prime}}, a_{m^{\prime}} q^{\lambda_{m}}}^{(m)}\right), & j=n .\end{cases}
$$

\section{Acknowledgements}

The author would like to thank Steven V. Sam for informing him of the results in [22]. This work was supported by JSPS Grant-in-Aid for Young Scientists (B) No. 25800006, and by World Premier International Research Center Initiative (WPI Initiative), MEXT, Japan.

\section{References}

[1] Chari V., Minimal affinizations of representations of quantum groups: the rank 2 case, Publ. Res. Inst. Math. Sci. 31 (1995), 873-911, hep-th/9410022.

[2] Chari V., On the fermionic formula and the Kirillov-Reshetikhin conjecture, Int. Math. Res. Not. 2001 (2001), 629-654, math.QA/0006090.

[3] Chari V., Braid group actions and tensor products, Int. Math. Res. Not. 2002 (2002), 357-382, math.QA/0106241.

[4] Chari V., Greenstein J., Minimal affinizations as projective objects, J. Geom. Phys. 61 (2011), 594-609, arXiv:1009.4494.

[5] Chari V., Moura A., The restricted Kirillov-Reshetikhin modules for the current and twisted current algebras, Comm. Math. Phys. 266 (2006), 431-454, math.RT/0507584.

[6] Chari V., Pressley A., A guide to quantum groups, Cambridge University Press, Cambridge, 1994.

[7] Chari V., Pressley A., Minimal affinizations of representations of quantum groups: the nonsimply-laced case, Lett. Math. Phys. 35 (1995), 99-114.

[8] Chari V., Pressley A., Quantum affine algebras and their representations, in Representations of Groups (Banff, AB, 1994), CMS Conf. Proc., Vol. 16, Amer. Math. Soc., Providence, RI, 1995, 59-78, hep-th/9411145.

[9] Chari V., Pressley A., Minimal affinizations of representations of quantum groups: the irregular case, Lett. Math. Phys. 36 (1996), 247-266.

[10] Chari V., Pressley A., Minimal affinizations of representations of quantum groups: the simply laced case, J. Algebra 184 (1996), 1-30, hep-th/9410036.

[11] Fourier G., Littelmann P., Tensor product structure of affine Demazure modules and limit constructions, Nagoya Math. J. 182 (2006), 171-198, math.RT/0412432.

[12] Hernandez D., On minimal affinizations of representations of quantum groups, Comm. Math. Phys. 276 (2007), 221-259, math.QA/0607527.

[13] Hernandez D., Leclerc B., Cluster algebras and quantum affine algebras, Duke Math. J. 154 (2010), 265-341, arXiv:0903.1452.

[14] Joseph A., On the Demazure character formula, Ann. Sci. École Norm. Sup. (4) 18 (1985), 389-419.

[15] Kac V.G., Infinite-dimensional Lie algebras, 3rd ed., Cambridge University Press, Cambridge, 1990. 
[16] Lakshmibai V., Littelmann P., Magyar P., Standard monomial theory for Bott-Samelson varieties, Compositio Math. 130 (2002), 293-318, alg-geom/9703020.

[17] Lusztig G., Introduction to quantum groups, Progress in Mathematics, Vol. 110, Birkhäuser Boston, Inc., Boston, MA, 1993.

[18] Moura A., Restricted limits of minimal affinizations, Pacific J. Math. 244 (2010), 359-397, arXiv:0812.2238.

[19] Mukhin E., Young C.A.S., Affinization of category $\mathcal{O}$ for quantum groups, Trans. Amer. Math. Soc., to appear, arXiv:1204.2769.

[20] Naoi K., Weyl modules, Demazure modules and finite crystals for non-simply laced type, Adv. Math. 229 (2012), 875-934, arXiv:1012.5480.

[21] Naoi K., Demazure modules and graded limits of minimal affinizations, Represent. Theory 17 (2013), 524556, arXiv:1210.0175.

[22] Sam S.V., Jacobi-Trudi determinants and characters of minimal affinizations, Pacific J. Math., to appear, arXiv:1307.6630. 